\title{
An integrable coupling hierarchy of Dirac integrable hierarchy, its Liouville integrability and Darboux transformation
}

\author{
Xi-Xiang $\mathrm{Xu}^{\mathrm{a}, *}$, Ye-Peng Sun ${ }^{\mathrm{b}}$ \\ ${ }^{a}$ College of Mathematics and Systems Science, Shandong University of Science and Technology, Qingdao, 266590, China. \\ ${ }^{b}$ School of Mathematics and Quantitative Economics, Shandong University of Finance and Economics, Jinan, 250014, China. \\ Communicated by $\mathrm{Y}$. Hu
}

\begin{abstract}
An integrable coupling hierarchy of Dirac integrable hierarchy is presented by means of zero curvature representation. A Hamiltonian operator involving two parameters is introduced, and it is used to derive a pair of Hamiltonian operators. A bi-Hamiltonian structure of the obtained integrable coupling hierarchy is constructed with the aid of Magri pattern of biHamiltonian formulation. Moreover, we prove the Liouville integrability of the obtained integrable coupling hierarchy and establish a Darboux transformation of the integrable coupling. As an application, an exact solution of the integrable coupling of Dirac equation is given. (c)2017 All rights reserved.
\end{abstract}

Keywords: Dirac integrable hierarchy, integrable coupling, Hamiltonian operator, Magri pattern, bi-Hamiltonian structure, Darboux transformation.

2010 MSC: 35Q58, 37K10.

\section{Introduction}

In recent years, the investigation of the integrable couplings of soliton equations has received considerable attention. The integrable couplings originate from the work on perturbations around solutions of evolution equations [18] and the perturbation bundle [1]. A few methods of constructing integrable couplings are proposed. Many problems of the integrable couplings have been studied $[3,6-12,20]$.

For a given integrable evolution equation

$$
\mathrm{u}_{\mathrm{t}}=\mathrm{K}(\mathrm{u}) \text {, }
$$

we actually want to construct a new bigger, triangular integrable system as follows:

$$
\left(\begin{array}{l}
u \\
v
\end{array}\right)_{t}=\left(\begin{array}{c}
K(u) \\
\chi(u, v)
\end{array}\right) .
$$

In Eq. (1.2), $\chi(u, v)$ is a real function defined over $R^{2}$, and it should satisfy the non-triviality condition $\frac{\partial \chi(u, v)}{\partial[u]} \neq 0$, where $[u]=\left(u, u_{x}, u_{x x}, \cdots\right)$. This statement means that the second equation in the

\footnotetext{
*Corresponding author

Email address: xixiang-xu@sohu.com (Xi-Xiang Xu)
}

doi:10.22436/jnsa.010.06.42 
bigger system (1.2) involves the dependent variable $u$ of the original integrable system (1.1). The Eq. (1.2) is called an integrable coupling of Eq. (1.1). In the theory of the integrable couplings, an important subject is to establish their bi-Hamiltonian structures. If an integrable coupling hierarchy possesses biHamiltonian structure, then we can derive a hereditary recursion operator and infinitely many conserved functionals. Thus, the Louville integrability of the integrable coupling hierarchy can be deduced. Usually, bi-Hamiltonian structures of integrable systems may be establish by using the trace identity [19]. However, it can not be used in the case of integrable couplings. We find that Magri pattern of bi-Hamiltonian formulation is very effective way to establish bi-Hamiltonian structure of the integrable hierarchy [13$15,17]$.

In addition, we know that Darboux transformation is a purely algebraic, powerful method to construct solutions of the integrable systems. The Lax pair plays a key role in the method of Darboux transformation $[2,16]$. To the best of our knowledge, in soliton theory, Darboux transformation of Lax pairs of the integrable couplings composed by triangular integrable system Eq. (1.2) has not been studied. In this paper, we are going to establish Darboux transformation of Lax pair for the integrable coupling of Dirac equation, which has the form of the Eq. (1.2).

It is well-known that the Dirac integrable system

$$
\left\{\begin{array}{l}
q_{t}=-\frac{1}{2} r_{x x}+q^{2} r+r^{3} \\
r_{t}=\frac{1}{2} q_{x x}-q r^{2}-q^{3},
\end{array}\right.
$$

is an important soliton equation. Its corresponding integrable hierarchy have been researched in Refs. $[3,5]$. In the following, we would like to research the integrable coupling of Dirac integrable system (1.3)

$$
\left\{\begin{array}{l}
q_{t}=-\frac{1}{2} r_{x x}+r q^{2}+r^{3} \\
r_{t}=\frac{1}{2} q_{x x}-q^{3}-q^{2} \\
s_{t}=-\frac{1}{2}\left(r_{x x}+w_{x x}\right)+(w+r)\left(q^{2}+r^{2}\right)+2 q r s+2 r^{2} w \\
w_{t}=\frac{1}{2}\left(q_{x x}+s_{x x}\right)-(q+s)\left(q^{2}+r^{2}\right)-2 q^{2} s-2 q r w .
\end{array}\right.
$$

Obviously, Eq. (1.4) has the form of the Eq. (1.2). In addition, if we set the Dirac integrable system (1.3) in the following form

$$
u_{\mathrm{t}}=\mathrm{K}(\mathrm{u}) \text {, }
$$

where $u=(q, r)^{\top}$, then the integrable coupling system (1.4) may be represented as

$$
\left\{\begin{array}{l}
u_{t}=K(u), \\
v_{t}=K(u)+K^{\prime}(u)[v] .
\end{array}\right.
$$

Here $v=(s, w)^{\top}, K^{\prime}(u)[v]$ denotes the Gateaux derivative of $K(u)$ with respect to $u$ in a direction $v$, i.e., $\mathrm{K}^{\prime}(u)[v]=\left.\frac{\partial}{\partial \varepsilon} \mathrm{K}(u+\varepsilon v)\right|_{\varepsilon=0}$. In view of perturbation theory [10], the integrable coupling (1.4) is first-order perturbation system of the Dirac integrable system (1.3).

This paper is organized as follows. In Section 2, we consider the spectral problem

$$
\varphi_{x}=U(u, \lambda) \varphi, U(u, \lambda)=\left(\begin{array}{cccc}
q & \lambda+r & s & w \\
-\lambda+r & -q & w & -s \\
0 & 0 & q & \lambda+r \\
0 & 0 & -\lambda+r & -q
\end{array}\right) .
$$

Locality of solution of the related stationary zero curvature equation is proved. An integrable coupling hierarchy of Dirac integrable hierarchy is deduced by using the zero curvature representation. In Section 3, a pair of Hamiltonian operators is presented by the aid of a Hamiltonian operator containing two arbitrary constants. A bi-Hamiltonian structure of obtained integrable coupling hierarchy is constructed by 
using the Magri pattern [13-15, 17]. Then Liouville integrability of the obtained integrable coupling hierarchy is demonstrated. In Section 4, a Darboux transformation of Lax pair of the Eq. (1.4) is established by means of a gauge transformation. In Section 5, as an application of Darboux transformation, an exact solution of the Eq. (1.4) is given. Finally, we give some conclusions and remarks.

\section{An integrable coupling hierarchy of Dirac integrable hierarchy}

First of all, we consider the stationary zero curvature equation associated with spectral problem (1.5)

$$
\mathrm{v}_{\mathrm{x}}=[\mathrm{U}, \mathrm{v}]=\mathrm{UV}-\mathrm{Vu},
$$

with

$$
V=V(u, \lambda)=\left(\begin{array}{cccc}
c & a+b & h & f+g \\
a-b & -c & f-g & -h \\
0 & 0 & c & a+b \\
0 & 0 & a-b & -c
\end{array}\right)
$$

The equation (2.1) implies

$$
\begin{aligned}
& c_{x}+2 r b-2 a \lambda=0 \\
& a_{x}+b_{x}-2(a+b) q+2 r c+2 c \lambda=0 \\
& h_{x}+2 r g+2 w b-2 f \lambda=0 \\
& f_{x}+g_{x}-2 q(f+g)-2 s(a+b)+2 r h+2 w c+2 h \lambda=0 \\
& a_{x}-b_{x}+2 q(a-b)-2 r c+2 c \lambda=0 \\
& f_{x}-g_{x}+2 q(f-g)+2 s(a-b)-2 r h-2 w c+2 h \lambda=0 .
\end{aligned}
$$

From (2.2), we get

$$
\begin{aligned}
2 \mathrm{a} \lambda & =2 r b+c_{x}, \\
b_{x} & =2 q a-2 r c, \\
2 c \lambda & =-a_{x}+2 q b, \\
2 f \lambda & =2 w b+2 r g+h_{x}, \\
g_{x} & =2 s a-2 w c+2 q f-2 r h, \\
2 h \lambda & =2 s b-f_{x}+2 q g .
\end{aligned}
$$

By substituting expansions

$$
a=\sum_{n=0}^{\infty} a_{n} \lambda^{-n}, b=\sum_{n=0}^{\infty} b_{n} \lambda^{-n}, c=\sum_{n=0}^{\infty} c_{n} \lambda^{-n}, f=\sum_{n=0}^{\infty} f_{n} \lambda^{-n}, g=\sum_{n=0}^{\infty} g_{n} \lambda^{-n}, h=\sum_{n=0}^{\infty} h_{n} \lambda^{-n},
$$

into (2.3), we obtain the initial conditions:

$$
a_{0}=0, b_{0 x}=2 q a_{0}-2 r c_{0}, c_{0}=0, f_{0}=0, g_{0 x}=2 s a_{0}-2 w c_{0}+2 q f_{0}-2 r h_{0}, h_{0}=0,
$$

and the recursion relations

$$
\begin{aligned}
a_{n+1} & =r b_{n}+\frac{1}{2} c_{n x}, \\
b_{n} & =\partial^{-1}\left(2 q a_{n}-2 r c_{n}\right), \\
c_{n+1} & =-\frac{1}{2} a_{n x}+q b_{n}, \\
f_{n+1} & =w b_{n}+r g_{n}+\frac{1}{2} h_{n x}, \\
g_{n} & =\partial^{-1}\left(2 s a_{n}-2 w c_{n}+2 q f_{n}-2 r h_{n}\right), \\
h_{n+1} & =s b_{n}+q g_{n}-\frac{1}{2} f_{n x},
\end{aligned}
$$


Next, we discuss the locality of solution of (2.1) (or (2.5)).

Proposition 2.1. In (2.4), if the initial values are selected as follows

$$
\mathrm{b}_{0}=1, \mathrm{~g}_{0}=1,
$$

then $a_{n}, b_{n}, c_{n}, f_{n}, g_{n}, h_{n}, n \geqslant 1$ which are solved by (2.5), are all local, and they only depend on $q, r, s, w$ and their spatial derivatives up to some finite order.

Proof. Using first and third equations in (2.5), we see that $a_{n+1}$ and $c_{n+1}$ can be determined locally by $a_{n}, b_{n}$, and $c_{n}, n \geqslant 0$. Similarly, from fourth and sixth equations in (2.5), we know that $f_{n+1}$ and $g_{n+1}$ can be determined locally by $a_{n}, b_{n}, c_{n}, f_{n}, g_{n}$ and $h_{n}, n \geqslant 0$. When $b_{n+1}$ and $g_{n+1}$ are derived from second and fifth equations in (2.5), we need to use operator $\partial^{-1}$ to solve the corresponding differential equations. Hereinafter, we are going to show that $b_{n+1}$ and $g_{n+1}$ may be deduced through an algebraic method rather than by solving the differential equations.

A direct verification can show

$$
\mathrm{V}^{2}=\left(\begin{array}{cccc}
\mathrm{a}^{2}-\mathrm{b}^{2}+\mathrm{c}^{2} & 0 & 2(\mathrm{af}-\mathrm{bg}+\mathrm{ch}) & 0 \\
0 & \mathrm{a}^{2}-\mathrm{b}^{2}+\mathrm{c}^{2} & 0 & 2(\mathrm{af}-\mathrm{bg}+\mathrm{ch}) \\
0 & 0 & \mathrm{a}^{2}-\mathrm{b}^{2}+\mathrm{c}^{2} & 0 \\
0 & 0 & 0 & \mathrm{a}^{2}-\mathrm{b}^{2}+\mathrm{c}^{2}
\end{array}\right)
$$

and

$$
\left(\mathrm{V}^{2}\right)_{\mathrm{x}}=\left[\mathrm{U}, \mathrm{V}^{2}\right]=0
$$

From above two equations, we have

$$
a^{2}-b^{2}+c^{2}=\gamma_{1}(t), \quad(a f-b g+c h)=\gamma_{2}(t),
$$

where $\gamma_{1}(t)$ and $\gamma_{2}(t)$ are arbitrary functions of time variable $t$ only. We then deduce two recursion relations for $b_{n+1}$ and $g_{n+1}$ :

$$
\begin{aligned}
& b_{n+1}=\frac{1}{2}\left(\sum_{j=1}^{n} a_{j} a_{n-j+1}-\sum_{j=1}^{n} b_{j} b_{n-j+1}+\sum_{j=1}^{n} c_{j} c_{n-j+1}-\gamma_{1}(t)\right), \\
& g_{n+1}=\sum_{j=1}^{n} a_{j} f_{n-j+1}-\sum_{j=1}^{n} b_{j} g_{n-j+1}+\sum_{j=1}^{n} c_{j} h_{n-j+1}-b_{n+1}-\gamma_{2}(t) .
\end{aligned}
$$

Further, we select $\gamma_{1}(t)=\gamma_{2}(t)=0$. Then, we obtain that $a_{n}, b_{n}, c_{n}, f_{n}, g_{n}, h_{n}, n \geqslant 1$ can be solved successively through the algebraic method. This completes the proof.

The first few terms are given by $a_{1}=r, b_{1}=0, c_{1}=q, f_{1}=(r+w), g_{1}=0, h_{1}=(q+s), a_{2}=$ $\frac{1}{2} q_{x}, b_{2}=\frac{1}{2}\left(q^{2}+r^{2}\right), c_{2}=-\frac{1}{2} r_{x}, f_{2}=\frac{1}{2}\left(s_{x}+q_{x}\right), g_{2}=(q s+r w)+\frac{1}{2} q^{2}+\frac{1}{2} r^{2}, h_{2}=-\frac{1}{2}\left(r_{x}+w_{x}\right), a_{3}=$ $-\frac{1}{4} r_{x x}+\frac{1}{2} r\left(q^{3}+r^{2}\right), b_{3}=\frac{1}{2} r q_{x},-\frac{1}{2} q r_{x}, c_{3}=-\frac{1}{4} q_{x x}+\frac{1}{2} q\left(q^{2}+r^{2}\right), f_{3}=-\frac{1}{4}\left(r_{x x}+w_{x x}\right)+\frac{1}{2}(w+r)\left(q^{2}+\right.$ $\left.r^{2}\right)+q r s+r^{2} w, g_{3}=\frac{1}{2}\left(w q_{x}-q w_{x}+r q_{x}-q r_{x}+r s_{x}-s r_{x}\right), h_{3}=-\frac{1}{4}\left(q_{x x}+s_{x x}\right)+\frac{1}{2}(q+s)\left(q^{2}+r^{2}\right)+$ $q^{2} s+q r w, \cdots$. Now we set

$$
V_{n}=\sum_{j=0}^{n}\left(\begin{array}{cccc}
c_{j} & a_{j}+b_{j} & h_{j} & f_{j}+g_{j} \\
a_{j}-b_{j} & -c_{j} & f_{j}-g_{j} & -h_{j} \\
0 & 0 & c_{j} & a_{j}+b_{j} \\
0 & 0 & a_{j}-b_{j} & -c_{j}
\end{array}\right) \lambda^{n-j} .
$$


To obtain the related integrable coupling hierarchy, we take the time evolution of the eigenfunction of the spectral problem (1.5) obeys the differential equation

$$
\varphi_{t_{n}}=V_{n} \varphi, n \geqslant 0 .
$$

Then the compatibility conditions of (1.5) and (2.6) are

$$
\mathrm{U}_{\mathrm{t}_{\mathrm{n}}}-\mathrm{V}_{\mathrm{nx}}+\left[\mathrm{U}, \mathrm{V}_{\mathrm{n}}\right]=0, \mathrm{n} \geqslant 0,
$$

which give rise to the following hierarchy of integrable equations

$$
\left\{\begin{array}{l}
q_{t_{n}}=2 a_{n+1} \\
r_{t_{n}}=-2 c_{n+1}, \quad n \geqslant 0 . \\
s_{t_{n}}=2 f_{n+1} \\
w_{t_{n}}=-2 h_{n+1}
\end{array}\right.
$$

Eq. (2.7) is the zero curvature representations of Eq. (2.8). The spectral problem (1.5) and the (2.6) compose Lax pairs of (2.8). It is easy to verify that the first nonlinear differential equation in (2.8), when $n=1$, under $t_{1}=t$, is the differential equation

$$
\left\{\begin{array}{l}
q_{t}=-\frac{1}{2} r_{x x}+r q^{2}+r^{3}, \\
r_{t}=\frac{1}{2} q_{x x}-q^{3}-q r^{2}, \\
s_{t}=-\frac{1}{2}\left(r_{x x}+w_{x x}\right)+(w+r)\left(q^{2}+r^{2}\right)+2 q r s+2 r^{2} w \\
w_{t}=\frac{1}{2}\left(q_{x x}+s_{x x}\right)-(q+s)\left(q^{2}+r^{2}\right)-2 q^{2} s-2 q r w .
\end{array}\right.
$$

Because first two differential equations in (2.9) form the Dirac integrable system (1.3), (2.9) is an integrable coupling of the Dirac integrable system (1.3). The time part of the Lax pair of the integrable coupling (2.9) may be given by

$$
\varphi_{\mathrm{t}}=V_{1} \varphi, \mathrm{V}_{1}=\left(\begin{array}{cccc}
\mathrm{V}_{11} & \mathrm{~V}_{12} & \mathrm{~V}_{13} & \mathrm{~V}_{14} \\
\mathrm{~V}_{21} & \mathrm{~V}_{22} & \mathrm{~V}_{23} & \mathrm{~V}_{24} \\
0 & 0 & \mathrm{~V}_{11} & \mathrm{~V}_{12} \\
0 & 0 & \mathrm{~V}_{21} & \mathrm{~V}_{22}
\end{array}\right)
$$

where $V_{11}=q \lambda-\frac{1}{2} r_{x}, V_{12}=\lambda^{2}+r \lambda+\frac{1}{2}\left(q_{x}+q^{2}+r^{2}\right), \quad V_{13}=(q+s) \lambda-\frac{1}{2}\left(r_{x}+w_{x}\right), \quad V_{14}=\lambda^{2}+r \lambda+$ $w \lambda+\frac{1}{2}\left(s_{x}+q_{x}\right)+(q s+r w)+\frac{1}{2} q^{2}+\frac{1}{2} r^{2}, \quad V_{21}=-\lambda^{2}+r \lambda+\frac{1}{2}\left(q_{x}-q^{2}-r^{2}\right), \quad V_{22}=-q \lambda+\frac{1}{2} r_{x}, \quad V_{23}=$ $-\lambda^{2}+(r+w) \lambda+\frac{1}{2}\left(s_{x}+g_{x}\right)-(q s+r w)-\frac{1}{2} q^{2}-\frac{1}{2} r^{2}, v_{24}=-(q+s) \lambda+\frac{1}{2}\left(r_{x}+w_{x}\right)$.

\section{A bi-Hamiltonian structure and Liouville integrability of the integrable coupling hierarchy (2.8)}

In this section, we are going to establish bi-Hamiltonian structure of the integrable coupling hierarchy (2.8). First, let us introduce some concepts for further discussion. The variational derivative and the inner product are defined respectively by

$$
\frac{\delta \tilde{H}}{\delta u}=\sum_{n \geqslant 0} \partial^{(-n)}\left(\frac{\partial H}{\partial u^{(n)}}\right), u^{(n)}=\partial^{n} u, \quad\langle\hat{f}, \hat{g}\rangle=\int(\hat{f}, \hat{g})_{R^{4}} d x,
$$

where $\hat{f}, \hat{g}$ are required to be rapidly vanished at the infinity, and $(\hat{f}, \hat{g})_{R^{4}}$ denotes the standard inner product of $\hat{f}$ and $\hat{g}$ in the Euclidean space $R^{4}$. Operator $J^{*}$ is defined by $\left\langle J^{*} \hat{f}, \hat{g}\right\rangle=\langle\hat{f}, J \hat{g}\rangle$, it is called adjoint operator of $J$ with respect to above inner product. If an operator has the property $J=-J^{*}$, then $J$ is 
called to be skew-symmetric. A linear operator J is called a Hamiltonian operator, if $\mathrm{J}$ is a skew-symmetric operator, and satisfies the Jacobi identity, i.e., it satisfies

$$
\left\langle J^{\prime}(u)[J \hat{f}] \hat{g}, \hat{h}\right\rangle+\operatorname{Cycle}(\hat{f}, \hat{g}, \hat{h})=0 .
$$

Based on a given Hamiltonian operator J, we can define a corresponding Poisson bracket $[4,9,17]$

$$
\{\hat{f}, \hat{g}\}_{J}=\left\langle\frac{\delta \hat{f}}{\delta u}, J \frac{\delta \hat{g}}{\delta u}\right\rangle=\int\left\langle\frac{\delta \hat{f}}{\delta u}, J \frac{\delta \hat{g}}{\delta u}\right\rangle_{R^{4}} d x
$$

If $J, M$ are Hamiltonian operators, and $\alpha J+\beta M$ is still a Hamiltonian operator for all real-values of $\alpha$ and $\beta$, then $J$ and $M$ form a pair of Hamiltonian operators. By means of the pair of Hamiltonian operators, we can obtain the bi-Hamiltonian structure of the related integrable system.

From the Eqs. (2.5) and Eq. (2.8), we get

$$
\left(\begin{array}{c}
2 a_{n+1} \\
-2 c_{n+1} \\
2 f_{n+1} \\
-2 h_{n+1}
\end{array}\right)=J\left(\begin{array}{c}
h_{n+1} \\
f_{n+1} \\
c_{n+1} \\
a_{n+1}
\end{array}\right)=M\left(\begin{array}{c}
h_{n} \\
f_{n} \\
c_{n} \\
a_{n}
\end{array}\right),
$$

where

$$
\begin{aligned}
J & =\left(\begin{array}{cccc}
0 & 0 & 0 & 2 \\
0 & 0 & -2 & 0 \\
0 & 2 & 0 & 0 \\
-2 & 0 & 0 & 0
\end{array}\right), \\
M & =\left(\begin{array}{cccc}
0 & 0 & \partial-4 r \partial^{-1} r & 4 r \partial^{-1} q \\
0 & 0 & 4 q \partial^{-1} r & \partial-4 q \partial^{-1} q \\
\partial-4 r \partial^{-1} r & 4 r \partial^{-1} q & -4 r \partial^{-1} w-4 w \partial^{-1} r & 4 r \partial^{-1} s+4 w \partial^{-1} q \\
4 q \partial^{-1} r & \partial-4 q \partial^{-1} q & 4 q \partial^{-1} w+4 s \partial^{-1} r & -4 q \partial^{-1} s-4 s \partial^{-1} q
\end{array}\right)
\end{aligned}
$$

Denote

$$
\Gamma(\alpha, \beta)=\alpha J+\beta M, \alpha, \beta, \in R
$$

Proposition 3.1. The operator $\Gamma(\alpha, \beta)$ is Hamiltonian operator for all values of two constants $\alpha$ and $\beta$.

Proof. It is easy to see that the operator $\Gamma(\alpha, \beta)$ is a skew-symmetric operator. Namely,

$$
\Gamma(\alpha, \beta)=-\Gamma(\alpha, \beta)^{*} .
$$

Moreover, we can prove that the operator $\Gamma(\alpha, \beta)$ satisfies the Jacobi identity (3.1). The concrete check is given in the appendix. The proof is completed.

Proposition 3.2. The operators J and M are all Hamiltonian operators, and they constitute a pair of Hamiltonian operator.

Proof. Obviously $\Gamma(1,0)=\mathrm{J}, \Gamma(0,1)=M, \alpha \mathrm{J}+\beta M=\Gamma(\alpha, \beta)$, thus, operators J and $M$ constitute a pair of Hamiltonian operators. The proof is completed. 
In addition, it is easy to verify that $\mathrm{J}$ is reversible, and

$$
\mathrm{J}^{-1}=\left(\begin{array}{cccc}
0 & 0 & 0 & \frac{1}{2} \\
0 & 0 & -\frac{1}{2} & 0 \\
0 & \frac{1}{2} & 0 & 0 \\
-\frac{1}{2} & 0 & 0 & 0
\end{array}\right)
$$

Setting

$$
\Psi=\mathrm{J}^{-1} \mathrm{M},
$$

and using the bi-Hamiltonian theory $[14,15,17]$, we know that its adjoint operator

$$
\Phi:=\Psi^{*}=\left(\begin{array}{cccc}
-2 r \partial^{-1} q & \frac{\partial}{2}-2 r \partial^{-1} r & 0 & 0 \\
-\frac{\partial}{2}+2 q \partial^{-1} q & 2 q \partial^{-1} r & 0 & 0 \\
-2 w \partial^{-1} q-2 r \partial^{-1} s & -2 w \partial^{-1} r-2 r \partial^{-1} w & -2 r \partial^{-1} q & \frac{\partial}{2}-2 r \partial^{-1} r \\
2 s \partial^{-1} q+2 q \partial^{-1} s & 2 s \partial^{-1} r+2 q \partial^{-1} w & -\frac{\partial}{2}+2 q \partial^{-1} q & 2 q \partial^{-1} r
\end{array}\right)
$$

is a hereditary operator.

Furthermore, from the Magri pattern, we can obtain the following bi-Hamiltonian initial equality

$$
J \frac{\delta \tilde{Y}_{2}}{\delta u}=M \frac{\delta \tilde{Y}_{1}}{\delta u}
$$

namely

$$
\frac{\delta \tilde{Y}_{2}}{\delta \mathrm{u}}=\Psi \frac{\delta \tilde{Y}_{1}}{\delta \mathrm{u}}
$$

where two Hamiltonian functionals are $\tilde{Y}_{i}=\int Y_{i}(u(x)) d x(1 \leqslant i \leqslant 2)$ and

$$
\mathrm{Y}_{1}=-(\mathrm{q} s+\mathrm{rw})+\frac{1}{2} \mathrm{q}^{2}+\frac{1}{2} \mathrm{r}^{2}, \mathrm{Y}_{2}=-\frac{1}{4}\left(w \mathrm{q}_{x}-\mathrm{q} w_{x}+r \mathrm{q}_{x}-\mathrm{qr}_{\mathrm{x}}+\mathrm{rs} \mathrm{s}_{\chi}-\mathrm{sr}_{\mathrm{x}}\right) .
$$

At present, from Eq. (2.8) and Eq. (3.3), we find that the integrable coupling hierarchy (2.8) can be rewritten as follows

$$
u_{t_{n}}=J \Psi^{n} y_{1}, n \geqslant 1,
$$

where $y_{1}:=\frac{\delta \tilde{Y}_{1}}{\delta u}=(q+s, r+w, q, r)^{\top}, y_{2}=\Psi y_{1}=\frac{\delta \tilde{Y}_{2}}{\delta u}$.

Note that J and $M$ constitute a pair of Hamiltonian operators. $y_{1}, y_{2}$ are gradients. According to the theory of bi-Hamiltonian operators, all vector functions $\Psi^{n} y_{1}(n \geqslant 1)$ are gradients [17]. Thus the equation hierarchy (3.5) (or (2.8)) has the following bi-Hamiltonian structure

$$
u_{t_{n}}=J \frac{\delta \tilde{Y}_{n}}{\delta u}=M \frac{\delta \tilde{Y}_{n-1}}{\delta u}, n>1,
$$

where the Hamiltonian functions $\tilde{Y}_{n}(n \geqslant 1)$ are given by

$$
\tilde{Y}_{n}=\int Y_{n}(u) d x, Y_{n}=\int_{0}^{1} u^{\top}\left(\Psi y_{1}\right)(\mu u) d \mu, n>1
$$

Proposition 3.3. The Hamiltonian functionals $\tilde{Y}_{n}(n \geqslant 1)$ are all common conserved functionals of the whole integrable coupling hierarchy (2.8) and commute with each other under the Poisson bracket (3.2). 
Proof. Because the operator $M$ is skew-symmetric, namely $M^{*}=-M$, then $J \Psi=\Phi J$. Therefore, we have

$$
\begin{aligned}
\left\{\tilde{Y}_{m}, \tilde{Y}_{l}\right\}_{J} & =\left\langle\frac{\delta \tilde{Y}_{m}}{\delta u}, J \frac{\delta \tilde{Y}_{l}}{\delta u}\right\rangle=\left\langle\Psi^{m-1} \frac{\delta \tilde{Y}_{1}}{\delta u}, J \Psi^{l-1} \frac{\delta \tilde{Y}_{1}}{\delta u}\right\rangle=\left\langle\Psi^{m-1} \frac{\delta \tilde{Y}_{1}}{\delta u}, \Psi^{*} J \Psi^{l-2} \frac{\delta \tilde{Y}_{1}}{\delta u}\right\rangle \\
& =\left\langle\Psi^{m} \frac{\delta \tilde{Y}_{1}}{\delta u}, J \Psi^{l-2} \frac{\delta \tilde{Y}_{1}}{\delta u}\right\rangle=\left\{\tilde{Y}_{m+1}, \tilde{Y}_{l-1}\right\}_{J}=\cdots=\left\{\tilde{Y}_{m+l-1}, \tilde{Y}_{1}\right\}_{J} .
\end{aligned}
$$

Similarly, we can also get

$$
\left\{\tilde{Y}_{l}, \tilde{Y}_{m}\right\}_{J}=\left\{\tilde{Y}_{m+l-1}, \tilde{Y}_{1}\right\}_{J}
$$

Thus, we have

$$
\left\{\tilde{Y}_{m}, \tilde{Y}_{l}\right\}_{J}=0, m, l \geqslant 1
$$

and

$$
\tilde{Y}_{n_{t_{m}}}=\left\langle\frac{\delta \tilde{Y}_{n}}{\delta u}, u_{n_{t_{m}}}\right\rangle=\left\langle\frac{\delta \tilde{Y}_{n}}{\delta u}, J \frac{\delta \tilde{Y}_{m}}{\delta u}\right\rangle=\left\{\tilde{Y}_{n}, \tilde{Y}_{m}\right\}_{J}=0, n, m \geqslant 1 .
$$

The proof is completed.

Based on Proposition 3.3, we can obtain the following theorem.

Theorem 3.4. The integrable couplings in (2.8) are all Liouville integrable bi-Hamiltonian systems.

\section{Darboux transformation}

It is well-known that Darboux transformations are a purely algebraic, powerful method to find explicit solutions of many soliton equations. Lax pair of the integrable system plays a key role $[2,16]$. We know that a gauge transformation of a matrix spectral problem is called Darboux transformation if it transforms the spectral problems into another spectral problems of the same type.

In what follows, we proceed to search for the Darboux transformation of the integrable coupling (1.4). We introduce a gauge transformation

$$
\tilde{\varphi}=\Pi^{(\mathrm{N})} \varphi .
$$

Here, we assume $\Pi^{(\mathrm{N})}$ is of the form

$$
\left(\begin{array}{cccc}
\sum_{i=0}^{N-1} A_{i} \lambda^{i} & \lambda^{N}+\sum_{i=0}^{N-1} B_{i} \lambda^{i} & \sum_{i=0}^{N-1} F_{i} \lambda^{i} & \lambda^{N}+\sum_{i=0}^{N-1} G_{i} \lambda^{i} \\
-\lambda^{N}+\sum_{i=0}^{N-1} C_{i} \lambda^{i} & \sum_{i=0}^{N-1} D_{i} \lambda^{i} & -\lambda^{N}+\sum_{i=0}^{N-1} H_{i} \lambda^{i} & \sum_{i=0}^{N-1} L_{i} \lambda^{i} \\
0 & 0 & \sum_{i=0}^{N-1} A_{i} \lambda^{i} & \lambda^{N}+\sum_{i=0}^{N-1} B_{i} \lambda^{i} \\
0 & 0 & -\lambda^{N}+\sum_{i=0}^{N-1} C_{i} \lambda^{i} & \sum_{i=0}^{N-1} D_{i} \lambda^{i}
\end{array}\right),
$$

where $N$ is a natural number, and $A_{i}, B_{i}, C_{i}, D_{i}, F_{i}, G_{i}, H_{i}, L_{i}, i=0,1, \cdots, N-1$ are undetermined functions of variables $x$ and t. Eq. (4.1) can transform two spectral problems (1.5) and (2.10) into

$$
\tilde{\varphi_{x}}=\tilde{U} \tilde{\varphi}, \quad \tilde{\varphi}_{t}=\tilde{V}_{1} \tilde{\varphi},
$$

where

$$
\tilde{\mathrm{U}}=\left(\left(\Pi^{(\mathrm{N})}\right)_{\mathrm{x}}+\Pi^{(\mathrm{N})} \mathrm{U}\right)\left(\Pi^{(\mathrm{N})}\right)^{-1}, \quad \tilde{\mathrm{V}}_{1}=\left(\left(\Pi^{(\mathrm{N})}\right)_{\mathrm{t}}+\Pi^{(\mathrm{N})} \mathrm{V}_{1}\right)\left(\Pi^{(\mathrm{N})}\right)^{-1} .
$$

In what follows, we determine $\Pi^{(N)}$ such that $\tilde{U}$ and $\tilde{V}_{1}$ in the Eq. (4.4) have the same form with $U$ and $V_{1}$, respectively. 
Let $\xi=\left(\xi_{1}, \xi_{2}, \xi_{3}, \xi_{4}\right)^{\top}, \eta=\left(\eta_{1}, \eta_{2}, \eta_{3}, \eta_{4}\right)^{\top}$ be two real linear independent solutions of Eq. (1.5) and Eq. (2.10), and use them to define the following four linear algebraic systems for $A_{i}, B_{i}, C_{i}, D_{i}$, $F_{i}, G_{i}, H_{i}$, and $L_{i}(1 \leqslant i \leqslant 2 N)$,

$$
\begin{gathered}
\sum_{i=0}^{N-1}\left(A_{i}+\alpha_{j} B_{i}+\beta_{j} F_{i}+\gamma_{i} G_{i}\right) \lambda_{j}^{i}=-\alpha_{j} \lambda_{j}^{N}-\gamma_{j} \lambda_{j}^{N}, \quad 1 \leqslant j \leqslant 2 N, \\
\sum_{i=0}^{N-1}\left(C_{i}+\alpha_{j} D_{i}+\beta_{j} H_{i}+\gamma_{i} L_{i}\right) \lambda_{j}^{i}=\lambda_{j}^{N}+\beta_{j} \lambda_{j}^{N}, \quad 1 \leqslant j \leqslant 2 N, \\
\sum_{i=0}^{N-1}\left(\beta_{j} A_{i}+\gamma_{j} B_{i}\right) \lambda_{j}^{i}=-\gamma_{j} \lambda_{j}^{N}, \quad 1 \leqslant j \leqslant 2 N, \\
\sum_{i=0}^{N-1}\left(\beta_{j} C_{i}+\gamma_{j} D_{i}\right) \lambda_{j}^{i}=\beta_{j} \lambda_{j}^{N}, \quad 1 \leqslant j \leqslant 2 N,
\end{gathered}
$$

with

$$
\alpha_{j}=\frac{\xi_{2}-\kappa_{j} \eta_{2}}{\xi_{1}-\kappa_{j} \eta_{1}}, \quad \beta_{j}=\frac{\xi_{3}-\kappa_{j} \eta_{3}}{\xi_{1}-\kappa_{j} \eta_{1}}, \quad \gamma_{j}=\frac{\xi_{4}-\kappa_{j} \eta_{4}}{\xi_{1}-\kappa_{j} \eta_{1}}, \quad j=1,2, \cdots, 2 N,
$$

and $\lambda_{j}, \kappa_{j}(1 \leqslant j \leqslant 2 N)$ are suitably chosen such that all the determinants of coefficients in $(4.5) \sim(4.9)$ are nonzero.

Therefore, $A_{i}, B_{i}, C_{i}, D_{i}, F_{i}, G_{i}, H_{i}$ and $L_{i}(0 \leqslant j \leqslant N-1)$ are uniquely determined. From (4.5) (4.8), it is easy to get that $\operatorname{det}\left(\Pi^{(N)}\right)$ is $4 N$ th-order polynomial of $\lambda$, and $\lambda_{j}(1 \leqslant j \leqslant 2 N)$ are all its roots. Thus, we have

$$
\operatorname{det}\left(\Pi^{(N)}\right)=\prod_{j=1}^{2 N}\left(\lambda-\lambda_{j}\right)^{2}
$$

Proposition 4.1. The matrix $\tilde{\mathrm{U}}$ defined by (4.4) has the same form as $\mathrm{U}$, in which the old potentials $\mathrm{q}, \mathrm{r}, \mathrm{s}$, and $w$ are mapped into new potentials $\tilde{\mathrm{q}}, \tilde{\mathrm{r}}, \tilde{\mathrm{s}}$, and $\tilde{\mathrm{w}}$ according to

$$
\left\{\begin{array}{l}
\tilde{q}=-q+A_{N-1}-D_{N-1} \\
\tilde{r}=-r+B_{N-1}+C_{N-1} \\
\tilde{s}=-s-A_{N-1}+D_{N-1}+F_{N-1}-L_{N-1} \\
\tilde{w}=-w-B_{N-1}-C_{N-1}+G_{N-1}+H_{N-1}
\end{array}\right.
$$

Proof. Let $A=\sum_{i=0}^{N-1} A_{i} \lambda^{i}, B=\lambda^{N}+\sum_{i=0}^{N-1} B_{i} \lambda^{i}, C=-\lambda^{N}+\sum_{i=0}^{N-1} C_{i} \lambda^{i}, D=\sum_{i=0}^{N-1} D_{i} \lambda^{i}, F=\sum_{i=0}^{N-1} F_{i} \lambda^{i}, G=$ $\lambda^{N}+\sum_{i=0}^{N-1} G_{i} \lambda^{i}, H=-\lambda^{N}+\sum_{i=0}^{N-1} H_{i} \lambda^{i}, L=\sum_{i=0}^{N-1} L_{i} \lambda^{i}$, and

$$
\left(\left(\Pi^{(\mathrm{N})}\right)_{x}+\Pi^{(\mathrm{N})} \mathrm{U}\right)\left(\Pi^{(\mathrm{N})}\right)^{*}=\left(\begin{array}{cccc}
\Gamma_{11}(\lambda) & \Gamma_{12}(\lambda) & \Gamma_{13}(\lambda) & \Gamma_{14}(\lambda) \\
\Gamma_{21}(\lambda) & \Gamma_{22}(\lambda) & \Gamma_{23}(\lambda) & \Gamma_{24}(\lambda) \\
0 & 0 & \Gamma_{11}(\lambda) & \Gamma_{12}(\lambda) \\
0 & 0 & \Gamma_{21}(\lambda) & \Gamma_{22}(\lambda)
\end{array}\right),
$$

where $\left(\Pi^{(N)}\right)^{-1}=\left(\Pi^{(N)}\right)^{*} / \operatorname{det}\left(\Pi^{(N)}\right)$. From (4.11), we have

$$
\begin{aligned}
& \Gamma_{11}(\lambda)=(A D-B C)\left(-B_{x} C+A_{x} D+q B C+q A D-r A C+r B D+A C \lambda-B D \lambda\right), \\
& \Gamma_{12}(\lambda)=(B C-A D)\left(A_{x} B-A\left(B_{x}-2 q B\right)+B^{2}(r-\lambda)-A^{2}(r+\lambda)\right),
\end{aligned}
$$




$$
\begin{aligned}
\Gamma_{13}(\lambda)= & (A D-B C)\left(D F_{x}-C G_{x}+q D F-q C G-r C F+r D G+s B C+s A D-w A C+w B D-C F \lambda-D G \lambda\right) \\
& +\left(B_{x}-q B+r A+A \lambda\right)\left(C D F-C^{2} G-A D H+A C L\right) \\
& +(A x+q A+r B+B \lambda)\left(-D^{2} F+C D G+B D H-B C L\right), \\
\Gamma_{14}(\lambda)= & (A D-B C)\left(-B F_{x}+A G_{x}-q B F-q A G+r A F-r B G-2 s A B+w A^{2}-w B^{2}+A F \lambda+B G \lambda\right) \\
& +\left(B_{x}-q B+r A+A \lambda\right)\left(-B C F+A C G+A B H-A^{2} L\right) \\
& +\left(A_{x}+q A+r B+B \lambda\right)\left(B D F-A D G-B^{2} H+A B L\right), \\
\Gamma_{21}(\lambda)= & (B C-A D)\left(-C_{x} D+C\left(D_{x}-2 q D\right)+D^{2}(-r+\lambda)+C^{2}(r+\lambda)\right), \\
\Gamma_{22}(\lambda)= & (B C-A D)\left(B\left(C_{x}+q C+D(r-\lambda)\right)-A\left(D_{x}-q D+C(r+\lambda)\right),\right. \\
\Gamma_{23}(\lambda)= & (A D-B C)\left(D H_{x}-C L_{x}+q D H-q C L-r C H+r D L+2 s C D-w C^{2}+w D^{2}-C H \lambda-D L \lambda\right) \\
& +\left(D_{x}-q D+r C+C \lambda\right)\left(C D F-C^{2} G-A D H+A C L\right) \\
& +\left(C_{x}+q C+r D-D \lambda\right)\left(-D^{2} F+C D G+B D H-B C L\right), \\
\Gamma_{24}(\lambda)= & (A D-B C)\left(A L_{x}-B H_{x}-q B H-q A L+r A H-r B L-s A D-s B C+w A C-w B D+A H \lambda+B L \lambda\right) \\
& +\left(D_{x}-q D+r C+C \lambda\right)\left(-B C F+A C G+A B H-A^{2} L\right) \\
& +\left(C_{x}+q C+r B-D \lambda\right)\left(B D F-A D G-B^{2} H+A B L\right) .
\end{aligned}
$$

We find that $\Gamma_{11}(\lambda), \Gamma_{13}(\lambda)$, and $\Gamma_{21}(\lambda)$ are $(4 N+1)$ th-order polynomials in $\lambda$, and $\Gamma_{12}(\lambda), \Gamma_{13}(\lambda), \Gamma_{14}(\lambda)$, $\Gamma_{21}(\lambda), \Gamma_{22}(\lambda)$, and $\Gamma_{24}(\lambda)$ are $4 \mathrm{~N}$ th-order polynomials in $\lambda$. Through a direct verify, we have

$$
\Gamma_{i j}\left(\lambda_{k}\right)=0, i=1,2, j=1,2,3,4, k=1, \cdots, 2 N .
$$

Then, we deduce

$$
\left(\left(\Pi^{(\mathrm{N})}\right)_{x}+\Pi^{(\mathrm{N})} \mathrm{U}\right)\left(\Pi^{(\mathrm{N})}\right)^{*}=\operatorname{det}\left(\Pi^{(\mathrm{N})}\right) \mathrm{M}
$$

with

$$
M=\left(\begin{array}{cccc}
m_{11}^{(0)} & m_{12}^{(1)} \lambda+m_{12}^{(0)} & m_{13}^{(0)} & m_{14}^{(0)} \\
m_{21}^{(1)} \lambda+m_{21}^{(0)} & m_{22}^{(0)} & m_{23}^{(0)} & m_{24}^{(0)} \\
0 & 0 & m_{11}^{(0)} & m_{12}^{(1)} \lambda+m_{12}^{(0)} \\
0 & 0 & m_{21}^{(1)} \lambda+m_{21}^{(0)} & m_{22}^{(0)}
\end{array}\right)
$$

where $m_{12}^{(1)}, m_{21}^{(1)}$, and $m_{i j}^{(0)}(i=1,2, j=1,2,3,4)$ are all independent of $\lambda$. Hence we get

$$
\left(\left(\Pi^{(\mathrm{N})}\right)_{\mathrm{x}}+\Pi^{(\mathrm{N})} \mathrm{U}\right)=\mathrm{M} \Pi^{(\mathrm{N})} .
$$

Equating the coefficients of $\lambda^{\mathrm{N}+\mathrm{i}},(i=0,1)$ in (4.12), we obtain that $m_{11}^{(0)}=-q+A_{N-1}-D_{N-1}=$ $\tilde{\mathrm{q}}, \mathrm{m}_{12}^{(1)}=1, \mathrm{~m}_{12}^{(0)}=-\mathrm{r}+\mathrm{B}_{\mathrm{N}-1}+\mathrm{C}_{\mathrm{N}-1}=\tilde{\mathrm{r}}, \mathrm{m}_{13}^{(0)}=-\mathrm{s}-\mathrm{A}_{\mathrm{N}-1}+\mathrm{D}_{\mathrm{N}-1}+\mathrm{F}_{\mathrm{N}-1}-\mathrm{L}_{\mathrm{N}-1}=\tilde{s}, \mathrm{~m}_{14}^{(0)}=$ $-w_{n}-B_{(N-1)}-C_{N-1}+G_{N-1}+H_{N-1}=\tilde{w}, m_{21}^{(1)}=-1, m_{21}^{(0)}=-r+B_{N-1}+C_{N-1}=\tilde{r}, m_{22}^{(0)}=q-$ $A_{N-1}+D_{N-1}=-\tilde{q}, m_{23}^{(0)}=-w_{n}-B_{N-1}-C_{N-1}+G_{N-1}+H_{N-1}=\tilde{w}, m_{24}^{(0)}=s+A_{N-1}-D_{N-1}-$ $\mathrm{F}_{\mathrm{N}-1}+\mathrm{L}_{\mathrm{N}-1}=-\tilde{\mathrm{s}}$. The proof is completed.

Proposition 4.2. The matrix $\tilde{V}_{1}$ defined by (4.4) has the same form as $\mathrm{V}_{1}$ under the transformation (4.10), i.e.,

$$
\tilde{V}_{1}=\left(\begin{array}{cccc}
\tilde{V}_{11} & \tilde{V}_{12} & \tilde{V}_{13} & \tilde{V}_{14} \\
\tilde{V}_{21} & \tilde{V}_{22} & \tilde{V}_{23} & \tilde{V}_{24} \\
0 & 0 & \tilde{V}_{11} & \tilde{V}_{12} \\
0 & 0 & \tilde{V}_{21} & \tilde{V}_{22}
\end{array}\right)
$$

where $\tilde{V}_{11}=\tilde{\mathrm{q}} \lambda-\frac{1}{2} \tilde{\mathrm{r}}_{x}, \tilde{\mathrm{V}}_{12}=\lambda^{2}+\tilde{\mathrm{r}} \lambda+\frac{1}{2}\left(\tilde{\mathrm{q}}_{x}+\tilde{\mathrm{q}}^{2}+\tilde{\mathrm{r}}^{2}\right), \tilde{\mathrm{V}}_{13}=(\tilde{\mathrm{q}}+\tilde{\mathrm{s}}) \lambda-\frac{1}{2}\left(\tilde{\mathrm{r}}_{x}+\tilde{w}_{x}\right), \tilde{\mathrm{V}}_{14}=\lambda^{2}+(\tilde{\mathrm{r}}+$ $\tilde{w}) \lambda+\frac{1}{2}\left(\tilde{s}_{x}+\tilde{q}_{x}\right)+(\tilde{\mathrm{q}} \tilde{s}+\tilde{\mathrm{r}} \tilde{\mathrm{w}})+\frac{1}{2} \tilde{\mathrm{q}}^{2}+\frac{1}{2} \tilde{\mathrm{r}}^{2}, \tilde{\mathrm{V}}_{21}=-\lambda^{2}+\tilde{\mathrm{r}} \lambda+\frac{1}{2}\left(\tilde{\mathrm{q}}_{x}-\tilde{\mathrm{q}}^{2}-\tilde{\mathrm{r}}^{2}\right), \tilde{\mathrm{V}}_{22}=-\tilde{\mathrm{q}} \lambda+\frac{1}{2} \tilde{\mathrm{r}}_{x}, \tilde{\mathrm{V}}_{23}=$ $-\lambda^{2}+(\tilde{\mathrm{r}}+\tilde{\mathfrak{w}}) \lambda+\frac{1}{2}\left(\tilde{s}_{x}+\tilde{\mathrm{q}}_{x}\right)-(\tilde{\mathrm{q}} \tilde{\mathrm{s}}+\tilde{\mathrm{r}} \tilde{\mathrm{w}})-\frac{1}{2}\left(\tilde{\mathrm{q}}^{2}+\tilde{\mathrm{r}}^{2}\right), \tilde{\mathrm{V}}_{24}=-(\tilde{\mathrm{q}}+\tilde{\mathrm{s}}) \lambda+\frac{1}{2}\left(\tilde{\mathrm{r}}_{x}+\tilde{w}_{x}\right)$. 
Proof. Let

$$
\left(\Pi_{\mathrm{t}}^{(\mathrm{N})}+\Pi^{(\mathrm{N})} V_{1}\right)\left(\Pi^{(\mathrm{N})}\right)^{*}=\left(\begin{array}{cccc}
\Sigma_{11}(\lambda) & \Sigma_{12}(\lambda) & \Sigma_{13}(\lambda) & \Sigma_{14}(\lambda) \\
\Sigma_{21}(\lambda) & \Sigma_{22}(\lambda) & \Sigma_{23}(\lambda) & \Sigma_{24}(\lambda) \\
0 & 0 & \Sigma_{11}(\lambda) & \Sigma_{12}(\lambda) \\
0 & 0 & \Sigma_{21}(\lambda) & \Sigma_{22}(\lambda)
\end{array}\right)
$$

We obtain that $\Sigma_{11}(\lambda), \Sigma_{13}(\lambda), \Sigma_{22}(\lambda)$, and $\Sigma_{24}(\lambda)$ are $(4 N+1)$ th-order polynomials in $\lambda$, and $\Sigma_{12}(\lambda)$, $\Sigma_{14}(\lambda), \Sigma_{21}(\lambda)$, and $\Sigma_{23}(\lambda)$ are $(4 N+2)$ th-order polynomials in $\lambda$. By a direct check, we get

$$
\Sigma_{i j}\left(\lambda_{k}\right)=0, i=1,2, j=1,2,3,4, k=1, \cdots, 2 N .
$$

Therefore, the following equation is established

$$
\left(\Pi_{\mathrm{t}}^{(\mathrm{N})}+\Pi^{(\mathrm{N})} \mathrm{V}_{1}\right)\left(\Pi^{(\mathrm{N})}\right)^{*}=\operatorname{det}\left(\Pi^{(\mathrm{N})}\right) \mathrm{Y}
$$

with

$$
\mathrm{Z}=\left(\begin{array}{cccc}
z_{11}^{(1)} \lambda+z_{11}^{(0)} & z_{12}^{(2)} \lambda^{2}+z_{12}^{(1)} \lambda+z_{12}^{(0)} & z_{13}^{(1)} \lambda+z_{13}^{(0)} & z_{14}^{(2)} \lambda^{2}+z_{14}^{(1)} \lambda+z_{14}^{(0)} \\
z_{21}^{(2)} \lambda^{2}+z_{21}^{(1)} \lambda+z_{21}^{(0)} & z_{22}^{(1)} \lambda+z_{22}^{(0)} & z_{23}^{(2)} \lambda^{2}+z_{23}^{(1)} \lambda+z_{23}^{(0)} & z_{24}^{(1)} \lambda+z_{24}^{(0)} \\
0 & 0 & z_{11}^{(1)} \lambda+z_{11}^{(0)} & z_{12}^{(2)} \lambda^{2}+z_{12}^{(1)} \lambda+z_{12}^{(0)} \\
0 & 0 & z_{21}^{(2)} \lambda^{2}+z_{21}^{(1)} \lambda+z_{21}^{(0)} & z_{22}^{(1)} \lambda+z_{22}^{(0)}
\end{array}\right)
$$

where $z_{12}^{(2)}, z_{14}^{(2)}, z_{21}^{(2)}, z_{23}^{(2)}, z_{i j}^{(k)},(i, j=1,2,3,4, k=0,1)$ are all independent of $\lambda$. Due to (4.13), we obtain

$$
\left(\Pi_{\mathrm{t}}^{(\mathrm{N})}+\Pi^{(\mathrm{N})} \mathrm{V}_{1}\right)=\mathrm{Y} \Pi^{(\mathrm{N})} .
$$

Comparing the coefficients of $\lambda$ in (4.14), we have

$$
\begin{aligned}
z_{11}^{(1)}= & -q+A_{N-1}-D_{N-1}=\tilde{q} \\
z_{11}^{(0)}= & -\frac{1}{2}\left(-r_{x}+B_{N-1, x}+C_{N-1, x}\right)=-\frac{1}{2} \tilde{r}_{x} \\
z_{12}^{(2)}= & 1 \\
z_{12}^{(1)}= & -r+B_{N-1}+C_{N-1}=\tilde{r} \\
z_{12}^{(0)}= & \frac{1}{2}\left(-q_{x}+A_{N-1, x}-D_{N-1, x}+\left(-q+A_{N-1}-D_{N-1}\right)^{2}+\left(-r+B_{N-1}+C_{N-1}\right)^{2}\right)=\frac{1}{2}\left(\tilde{q}_{x}+\tilde{q}^{2}+\tilde{r}^{2}\right), \\
z_{13}^{(1)}= & \left(-q+A_{N-1}-D_{N-1}\right)+\left(-s-A_{N-1}+D_{N-1}+F_{N-1}-L_{N-1}\right)=(\tilde{q}+\tilde{s}), \\
z_{13}^{(0)}= & -\frac{1}{2}\left(-r_{x}+B_{N-1, x}+C_{N-1, x}\right)+\left(-w_{x}-B_{N-1, x}-C_{N-1, x}+G_{N-1, x}+H_{N-1, x}\right)=-\frac{1}{2}\left(\tilde{r}_{x}+\tilde{w}_{x}\right), \\
z_{14}^{(2)}= & 1 \\
z_{14}^{(1)}= & \left(-r_{x}+B_{N-1, x}+C_{N-1, x}\right)+\left(-w_{x}-B_{N-1, x}-C_{N-1, x}+G_{N-1, x}+H_{N-1, x}\right)=\tilde{r}_{x}+\tilde{w}_{x}, \\
z_{14}^{(0)}= & \frac{1}{2}\left(-s_{x}-A_{N-1, x}+D_{N-1, x}+F_{N-1, x}-L_{N-1, x}\right) \\
& +\left(-q_{x}+A_{N-1, x}-D_{N-1, x}\right)+\left(-q+A_{N-1}-D_{N-1}\right)\left(-s-A_{N-1}+D_{N-1}+F_{N-1}-L_{N-1}\right) \\
& \left.+\left(-r+B_{N-1}+C_{N-1}\right)\left(-w-B_{N-1}-C_{N-1}+G_{N-1}+H_{N-1}\right)\right) \\
& +\frac{1}{2}\left(\left(-q+A_{N-1}-D_{N-1}\right)^{2}+\left(-r+B_{N-1}+C_{N-1}\right)^{2}\right)=\frac{1}{2}\left(\tilde{s}_{x}+\tilde{g}_{x}\right)+(\tilde{q} \tilde{s}+\tilde{r} \tilde{w})+\frac{1}{2}\left(\tilde{q}^{2}+\tilde{r}^{2}\right), \\
z_{21}^{(2)}= & -1,
\end{aligned}
$$




$$
\begin{aligned}
z_{21}^{(1)}= & -r+B_{N-1}+C_{N-1}=\tilde{r}, \\
z_{21}^{(0)}= & \frac{1}{2}\left(-q_{x}+A_{N-1, x}-D_{N-1, x}-\left(-q+A_{N-1}-D_{N-1}\right)^{2}-\left(-r+B_{N-1}+C_{N-1}\right)^{2}\right)=\frac{1}{2}\left(\tilde{q}_{x}-\tilde{q}^{2}-\tilde{r}^{2}\right), \\
z_{22}^{(1)}= & -\left(-q+A_{N-1}-D_{N-1}\right)=-\tilde{q} \\
z_{22}^{(0)}= & \frac{1}{2}\left(-r_{x}+B_{N-1, x}+C_{N-1, x}\right)=\frac{1}{2} \tilde{r}_{x}, x_{23}^{(2)}=-1, \\
z_{23}^{(1)}= & \left(-r+B_{N-1}+C_{N-1}+\left(-w-B_{N-1}-C_{N-1}+G_{N-1}+H_{N-1}\right)\right)=\tilde{r}+\tilde{w}, \\
z_{23}^{(0)}= & \frac{1}{2}\left(\left(-s_{x}-A_{N-1, x}+D_{N-1, x}+F_{N-1, x}-L_{N-1, x}\right)+\left(-q_{x}+A_{N-1, x}-D_{N-1, x}\right)\right) \\
& -\left(\left(-q+A_{N-1}-D_{N-1}\right)\left(-s-A_{N-1}+D_{N-1}+F_{N-1}-L_{N-1}\right)+\left(-r+B_{N-1}\right.\right. \\
& \left.\left.+C_{N-1}\right)\left(-w-B_{N-1}-C_{N-1}+G_{N-1}+H_{N-1}\right)\right)-\frac{1}{2}\left(\left(-q+A_{N-1}-D_{N-1}\right)^{2}\right. \\
& \left.+\left(-r+B_{N-1}+C_{N-1}\right)^{2}\right)=\frac{1}{2}\left(\tilde{s}_{x}+\tilde{q}_{x}\right)-(\tilde{q} \tilde{s}+\tilde{r} \tilde{w})-\frac{1}{2}\left(\tilde{q}^{2}+\tilde{r}^{2}\right), \\
z_{24}^{(1)}= & \left.-\left(-q+A_{N-1}-D_{N-1}\right)+\left(-s-A_{N-1}+D_{N-1}+F_{N-1}-L_{N-1}\right)=(\tilde{q}+\tilde{s})\right), \\
z_{24}^{(0)}= & \frac{1}{2}\left(\left(-r_{x}+B_{N-1, x}+C_{N-1, x}\right)+\left(-w_{x}-B_{N-1, x}-C_{N-1, x}+G_{N-1, x}+H_{N-1, x}\right)\right)=\frac{1}{2}\left(\tilde{r}_{x}+\tilde{w}_{x}\right) .
\end{aligned}
$$

The proof is completed.

Hence, we conclude that the transformations (4.3) and (4.4) can change the Lax pair Eq. (1.5) and Eq. (2.10) into another Lax pair with same form. Thus both of the Lax pairs lead to the same integrable coupling (1.4).

As usual, the gauge transformation (4.3), (4.4), and (4.10),

$$
(\varphi ; q, r, s, w)->\left(\tilde{\varphi}_{n}, \tilde{\mathrm{q}}, \tilde{\mathrm{r}}, \tilde{\mathrm{s}}, \tilde{w}\right)
$$

is a Darboux transformation of (1.5) and (2.10). Eq. (4.10) is so-called a Bäcklund transformation (BT) between new solution $(\tilde{\mathrm{q}}, \tilde{\mathrm{r}}, \tilde{\mathrm{s}}, \tilde{\mathrm{w}})^{\top}$ and old solution $(\mathrm{q}, \mathrm{r}, \mathrm{s}, \boldsymbol{w})^{\top}$.

In conclusion, according to propositions 4.1 and 4.2 , we have the following theorem.

Theorem 4.3. Each solution $(\mathrm{q}, r, s, w)^{\top}$ of (1.4) is mapped into new solution $(\tilde{\mathrm{q}}, \tilde{\mathrm{r}}, \tilde{\mathrm{s}}, \tilde{\mathrm{w}})^{\top}$ under the transformation (4.10).

\section{Explicit solution}

In what follows, we will apply Darboux transformation (4.15) to give explicit solution of (1.4). We choose a seed solution of (1.4) (i.e., a simple special solution) $(q, r, s, w)^{\top}=(0,0,1,1)^{\top}$ and $N=1$ in (4.2). Substituting this solution into the (1.5) and (2.10), we can obtain the following Lax pair

$$
\varphi_{x}=\left(\begin{array}{cccc}
0 & \lambda & 1 & 1 \\
-\lambda & 0 & 1 & -1 \\
0 & 0 & 0 & \lambda \\
0 & 0 & -\lambda & 0
\end{array}\right) \varphi, \quad \varphi_{\mathrm{t}}=\left(\begin{array}{cccc}
0 & \lambda & 1 & \lambda+1 \\
-\lambda & 0 & -\lambda+1 & -1 \\
0 & 0 & 0 & \lambda \\
0 & 0 & -\lambda & 0
\end{array}\right) \varphi .
$$

Solving above two equations, we have

$$
\phi=\left(\begin{array}{c}
\lambda t \cos (\lambda(x+t))+\frac{\sin (\lambda(x+t))}{\lambda} \\
-\lambda t \sin (\lambda(x+t))-\frac{\sin (\lambda(x+t))}{\lambda} \\
\sin (\lambda(x+t)) \\
\cos (\lambda(x+t))
\end{array}\right), \psi=\left(\begin{array}{c}
\lambda t \sin (\lambda(x+t))-\frac{\sin (\lambda(x+t))}{\lambda} \\
\lambda t \cos (\lambda(x+t))-\frac{\sin (\lambda(x+t))}{\lambda} \\
-\cos (\lambda(x+t)) \\
\sin (\lambda(x+t))
\end{array}\right)
$$




$$
\begin{gathered}
\alpha_{j}=\frac{\lambda_{j} t\left(-\sin \left(\lambda_{j}(x+t)\right)-\kappa_{j} \cos \left(\lambda_{j}(x+t)\right)+\left(-1+\kappa_{j}\right) \frac{\sin \left(\lambda_{j}(x+t)\right)}{\lambda_{j}}\right.}{\lambda_{j} t\left(\cos \left(\lambda_{j}(x+t)\right)-\kappa_{j} \sin \left(\lambda_{j}(x+t)\right)+\left(1+\kappa_{j}\right) \frac{\sin \left(\lambda_{j}(x+t)\right)}{\lambda_{j}}\right.}, \quad j=1,2, \\
\beta_{j}=\frac{\sin \left(\lambda_{j}(x+t)\right)+\kappa_{j} \cos \left(\lambda_{j}(x+t)\right)}{\lambda_{j} t\left(\cos \left(\lambda_{j}(x+t)\right)-\kappa_{j} \sin \left(\lambda_{j}(x+t)\right)+\left(1+\kappa_{j}\right) \frac{\sin \left(\lambda_{j}(x+t)\right)}{\lambda_{j}}\right.}, \quad j=1,2, \\
\gamma_{j}=\frac{\cos \left(\lambda_{j}(x+t)\right)-\kappa_{j} \sin \left(\lambda_{j}(x+t)\right)}{\lambda_{j} t\left(\cos \left(\lambda_{j}(x+t)\right)-\kappa_{j} \sin \left(\lambda_{j}(x+t)\right)+\left(1+\kappa_{j}\right) \frac{\sin \left(\lambda_{j}(x+t)\right)}{\lambda_{j}}\right.}, \quad j=1,2 .
\end{gathered}
$$

The Eq. (4.2) becomes

$$
\Pi^{(1)}=\left(\begin{array}{cccc}
A_{0} & \lambda+B_{0} & F_{0} & \lambda+G_{0} \\
-\lambda+C_{0} & D_{0} & -\lambda+H_{0} & L_{0} \\
0 & 0 & A_{0} & \lambda+B_{0} \\
0 & 0 & -\lambda+C_{0} & D_{0}
\end{array}\right)
$$

From Eq. (4.5) Eq. (4.9), we have

$$
\begin{aligned}
A_{0}= & \frac{\gamma_{1} \gamma_{2} \lambda_{2}-\gamma_{1} \gamma_{2} \lambda_{1}}{\beta_{1} \gamma_{2}-\beta_{2} \gamma_{1}}, B_{0}=\frac{\beta_{1} \gamma_{2} \lambda_{2}-\beta_{2} \gamma_{1} \lambda_{1}}{\beta_{2} \gamma_{1}-\beta_{1} \gamma_{2}}, C_{0}=\frac{\beta_{1} \gamma_{2} \lambda_{1}-\beta_{2} \gamma_{1} \lambda_{2}}{\beta_{1} \gamma_{2}-\beta_{2} \gamma_{1}}, D_{0}=\frac{\beta_{1} \beta_{2} \lambda_{1}-\beta_{1} \beta_{2} \lambda_{2}}{\beta_{1} \gamma_{2}-\beta_{2} \gamma_{1}}, \\
F_{0}= & \frac{\lambda_{1}-\lambda_{2}}{\left(\beta_{2} \gamma_{1}-\beta_{1} \gamma_{2}\right)^{2}}\left(\alpha_{2} \beta_{2} \gamma_{1}^{2}-\gamma_{2}\left(\alpha_{1} \beta_{1} \gamma_{2}+\gamma_{1}\left(\gamma_{1}-\beta_{2} \gamma_{1}+\left(-1+\beta_{1}\right) \gamma_{2}\right)\right)\right), \\
G_{0}= & \frac{1}{\left(\beta_{2} \gamma_{1}-\beta_{1} \gamma_{2}\right)^{2}}\left(-\beta_{2}^{2} \gamma_{1}^{2} \lambda_{1}+\alpha_{2} \beta_{1} \beta_{2} \gamma_{1}\left(-\lambda_{1}+\lambda_{2}\right)+\beta_{1} \gamma_{2}\left(\gamma_{1} \lambda_{1}-\left(\gamma_{1}+\beta_{1} \gamma_{2}\right) \lambda_{2}\right)\right. \\
& \left.+\beta_{2} \gamma_{2}\left(\alpha_{1} \beta_{1}\left(\lambda_{1}-\lambda_{2}\right)+\gamma_{1}\left(\left(-1+\beta_{1}\right) \lambda_{1}+\lambda_{2}+\beta_{1} \lambda_{2}\right)\right)\right), \\
\mathrm{H}_{0}= & \frac{1}{\left(\beta_{2} \gamma_{1}-\beta_{1} \gamma_{2}\right)^{2}}\left(\beta_{1}^{2} \gamma_{2}^{2} \lambda_{1}+\alpha_{1} \beta_{1} \beta_{2} \gamma_{2}\left(\lambda_{1}-\lambda_{2}\right)+\alpha_{2} \beta_{1} \beta_{2} \gamma_{1}\left(-\lambda_{1}+\lambda_{2}\right)-\beta_{1} \gamma_{1} \gamma_{2}\left(\left(-1+\beta_{2}\right) \lambda_{1}+\lambda_{2}\right.\right. \\
& \left.\left.+\beta_{2} \lambda_{2}\right)+\beta_{2} \gamma_{1}\left(\beta_{2} \gamma_{1} \lambda_{2}+\gamma_{2}\left(-\lambda_{1}+\lambda_{2}\right)\right)\right), \\
\mathrm{L}^{(0)}= & \frac{\lambda_{1}-\lambda_{2}}{\left(\beta_{2} \gamma_{1}-\beta_{1} \gamma_{2}\right)^{2}}\left(\alpha_{2} \beta_{1}^{2} \beta_{2}+\beta_{2}^{2}\left(-\alpha_{1} \beta_{1}+\gamma_{1}+\beta_{1} \gamma_{1}\right)-\beta_{1}^{2}\left(1+\beta_{2}\right) \gamma_{2}\right) .
\end{aligned}
$$

Eq. (4.10) becomes

$$
\left\{\begin{array}{l}
\tilde{q}=-q+A_{0}-D_{0}, \\
\tilde{r}=-r+B_{0}+C_{0} \\
\tilde{s}=-s-A_{0}+D_{0}+F_{0}-L_{0} \\
\tilde{w}=-w-B_{0}-C_{0}+G_{0}+H_{0} .
\end{array}\right.
$$

By the aid of Darboux transformation (4.15), we obtain new explicit solution of (1.4):

$$
\begin{aligned}
\tilde{\mathrm{q}}= & \frac{\gamma_{1} \gamma_{2} \lambda_{2}-\gamma_{1} \gamma_{2} \lambda_{1}}{\beta_{1} \gamma_{2}-\beta_{2} \gamma_{1}}-\frac{\beta_{1} \beta_{2} \lambda_{1}-\beta_{1} \beta_{2} \lambda_{2}}{\beta_{1} \gamma_{2}-\beta_{2} \gamma_{1}}, \quad \tilde{r}=\frac{\beta_{1} \gamma_{2} \lambda_{2}-\beta_{2} \gamma_{1} \lambda_{1}}{\beta_{2} \gamma_{1}-\beta_{1} \gamma_{2}}+\frac{\beta_{1} \gamma_{2} \lambda_{1}-\beta_{2} \gamma_{1} \lambda_{2}}{\beta_{1} \gamma_{2}-\beta_{2} \gamma_{1}}, \\
\tilde{s}= & -1-\frac{\gamma_{1} \gamma_{2} \lambda_{2}-\gamma_{1} \gamma_{2} \lambda_{1}}{\beta_{1} \gamma_{2}-\beta_{2} \gamma_{1}}+\frac{\beta_{1} \beta_{2} \lambda_{1}-\beta_{1} \beta_{2} \lambda_{2}}{\beta_{1} \gamma_{2}-\beta_{2} \gamma_{1}}+\frac{\lambda_{1}-\lambda_{2}}{\left(\beta_{2} \gamma_{1}-\beta_{1} \gamma_{2}\right)^{2}}\left(\alpha_{2} \beta_{2} \gamma_{1}^{2}-\gamma_{2}\left(\alpha_{1} \beta_{1} \gamma_{2}+\gamma_{1}\left(\gamma_{1}-\beta_{2} \gamma_{1}\right.\right.\right. \\
& \left.\left.\left.+\left(-1+\beta_{1}\right) \gamma_{2}\right)\right)\right)-\frac{\lambda_{1}-\lambda_{2}}{\left(\beta_{2} \gamma_{1}-\beta_{1} \gamma_{2}\right)^{2}}\left(\alpha_{2} \beta_{1}^{2} \beta_{2}+\beta_{2}^{2}\left(-\alpha_{1} \beta_{1}+\gamma_{1}+\beta_{1} \gamma_{1}\right)-\beta_{1}^{2}\left(1+\beta_{2}\right) \gamma_{2}\right), \\
\tilde{w}= & -1-\frac{\beta_{1} \gamma_{2} \lambda_{2}-\beta_{2} \gamma_{1} \lambda_{1}}{\beta_{2} \gamma_{1}-\beta_{1} \gamma_{2}}-\frac{\beta_{1} \gamma_{2} \lambda_{1}-\beta_{2} \gamma_{1} \lambda_{2}}{\beta_{1} \gamma_{2}-\beta_{2} \gamma_{1}}+\frac{1}{\left(\beta_{2} \gamma_{1}-\beta_{1} \gamma_{2}\right)^{2}}\left(-\beta_{2}^{2} \gamma_{1}^{2} \lambda_{1}+\alpha_{2} \beta_{1} \beta_{2} \gamma_{1}\left(-\lambda_{1}+\lambda_{2}\right)\right. \\
& \left.+\beta_{1} \gamma_{2}\left(\gamma_{1} \lambda_{1}-\left(\gamma_{1}+\beta_{1} \gamma_{2}\right) \lambda_{2}\right)+\beta_{2} \gamma_{2}\left(\alpha_{1} \beta_{1}\left(\lambda_{1}-\lambda_{2}\right)+\gamma_{1}\left(\left(-1+\beta_{1}\right) \lambda_{1}+\lambda_{2}+\beta_{1} \lambda_{2}\right)\right)\right)
\end{aligned}
$$




$$
\begin{aligned}
& +\frac{1}{\left(\beta_{2} \gamma_{1}-\beta_{1} \gamma_{2}\right)^{2}}\left(\beta_{1}^{2} \gamma_{2}^{2} \lambda_{1}+\alpha_{1} \beta_{1} \beta_{2} \gamma_{2}\left(\lambda_{1}-\lambda_{2}\right)+\alpha_{2} \beta_{1} \beta_{2} \gamma_{1}\left(-\lambda_{1}+\lambda_{2}\right)\right. \\
& \left.-\beta_{1} \gamma_{1} \gamma_{2}\left(\left(-1+\beta_{2}\right) \lambda_{1}+\lambda_{2}+\beta_{2} \lambda_{2}\right)+\beta_{2} \gamma_{1}\left(\beta_{2} \gamma_{1} \lambda_{2}+\gamma_{2}\left(-\lambda_{1}+\lambda_{2}\right)\right)\right)
\end{aligned}
$$

\section{Conclusions and remarks}

Starting from a four-by-four matrix spectral problem, we derived an integrable coupling hierarchy of Dirac integrable hierarchy by means of zero curvature equation. A bi-Hamiltonian structure of the obtained integrable coupling hierarchy was established by the Magri pattern of bi-Hamiltonian formulation. A hereditary operator of the obtained integrable coupling hierarchy was presented. Liouville integrability of the obtained Hamiltonian systems is demonstrated. With the help of a gauge transformation of the Lax pair, we establish a Darboux transformation of the Lax pair of obtained integrable coupling (1.4). Ultimately, as an application of the Darboux transformation, an explicit solution of the resulting integrable coupling is given.

From the obtained explicit solution, we apply the Darboux transformation (4.15) once again, then other new solution of Eq. (1.4) is obtained. This process can be done continually. Therefore, we can obtain a lot of explicit solutions for the resulting integrable coupling system of the Dirac integrable system.

In addition, many interesting problems deserve further investigation for the integrable coupling hierarchy (2.8). For example, inverse scattering transformation, nonlinearization of Lax pairs, constructing complexion solutions by the Wronskian determinant, conservation laws, and so on.

\section{Appendix: The proof of the Jacobi identity in Proposition 3.1}

We would like to give a detailed check of the Jacobi identity

$$
\left\langle\Gamma(\alpha, \beta)^{\prime}(u)[\Gamma(\alpha, \beta) f] g, h\right\rangle+\operatorname{Cycle}(f, g, h)=0,
$$

in which the operator $\Gamma(\alpha, \beta)$ is defined by (3.4). $u=(q, r, s, w)^{\top}$

$$
\hat{f}=\left(\hat{f}_{1}, \hat{f}_{2}, \hat{f}_{3}, \hat{f}_{4}\right)^{\top}, \hat{g}=\left(\hat{g}_{1}, \hat{g}_{2}, \hat{g}_{3}, \hat{g}_{4}\right)^{\top}, \hat{h}=\left(\hat{h}_{1}, \hat{h}_{2}, \hat{h}_{3}, \hat{h}_{4}\right)^{\top},
$$

where $\hat{f}_{i}=\hat{f}_{i}(x, t), \hat{g}_{i}=\hat{g}_{i}(x, t), \hat{h}_{i}=\hat{h}_{i}(x, t), i=1,2,3,4$. They are arbitrary functions, which are required to be rapidly vanishing at the infinity. We combine the terms in $\left\langle\Gamma(\alpha, \beta)^{\prime}(u)[\Gamma(\alpha, \beta) \hat{f}] \hat{g}, \hat{h}\right\rangle+$ Cycle $(\hat{f}, \hat{g}, \hat{h})$ containing $\alpha \beta, \beta^{2}$ respectively. Note that

$$
\left(\partial^{-1}\right)^{*}=-\partial^{-1},\left(\partial \hat{f}_{i}\right)\left(\partial^{-1} \hat{g}_{j}\right) \hat{h}_{k}=\hat{f}_{i} \hat{g}_{j}\left(\partial^{-1} \hat{h}_{k}\right), i, j, k=1,2,3,4 .
$$

Through a tediously but direct computation, the coefficients of $\alpha \beta, \beta^{2}$ can be obtained as follows.

$$
\begin{aligned}
\alpha \beta: 8 & \left\{\left\{\hat{f}_{3}\left(\partial^{-1}\left(r \hat{g}_{3}\right)\right) \hat{h}_{1}-\hat{f}_{3} \hat{g}_{3}\left(\partial^{-1}\left(r \hat{h}_{1}\right)\right)-\hat{f}_{3}\left(\partial^{-1}\left(q \hat{g}_{4}\right)\right) \hat{h}_{1}-\hat{f}_{4} \hat{g}_{4}\left(\partial^{-1}\left(r \hat{h}_{1}\right)\right)+\hat{f}_{4}\left(\partial^{-1}\left(r \hat{g}_{3}\right)\right) \hat{h}_{2}\right.\right. \\
& +\hat{f}_{3} \hat{g}_{3}\left(\left(\partial^{-1}\left(q \hat{h}_{2}\right)\right)-\hat{f}_{4}\left(\partial^{-1}\left(q \hat{g}_{4}\right)\right) \hat{h}_{2}+\hat{f}_{4} \hat{g}_{4}\left(\partial^{-1}\left(q \hat{h}_{2}\right)\right)+\hat{f}_{3}\left(\partial^{-1}\left(r \hat{g}_{1}\right)\right) \hat{h}_{3}-\hat{f}_{3} \hat{g}_{1}\left(\partial^{-1}\left(r \hat{h}_{3}\right)\right)\right. \\
& -\hat{f}_{3}\left(\partial^{-1}\left(q \hat{g}_{2}\right)\right) \hat{h}_{3}-\hat{f}_{4} \hat{g}_{2}\left(\partial^{-1}\left(r \hat{h}_{3}\right)\right)+\hat{f}_{3}\left(\partial^{-1}\left(w \hat{g}_{3}\right)\right) \hat{h}_{3}-\hat{f}_{1} \hat{g}_{3}\left(\partial^{-1}\left(r \hat{h}_{3}\right)\right)+\hat{f}_{1}\left(\partial^{-1}\left(r \hat{g}_{3}\right)\right) \hat{h}_{3} \\
& -\hat{f}_{3} \hat{g}_{3}\left(\partial^{-1}\left(w \hat{h}_{3}\right)\right)-\hat{f}_{2} \hat{g}_{4}\left(\partial^{-1}\left(r \hat{h}_{3}\right)\right)-\hat{f}_{3}\left(\partial^{-1}\left(s \hat{g}_{4}\right)\right) \hat{h}_{3}-\hat{f}_{4} \hat{g}_{4}\left(\partial^{-1}\left(w \hat{h}_{3}\right)\right)-\hat{f}_{1}\left(\partial^{-1}\left(q \hat{g}_{4}\right)\right) \hat{h}_{3} \\
& +\hat{f}_{4}\left(\partial^{-1}\left(r \hat{g}_{1}\right)\right) \hat{h}_{4}+\hat{f}_{3} \hat{g}_{1}\left(\partial^{-1}\left(q \hat{h}_{4}\right)\right)-\hat{f}_{4}\left(\partial^{-1}\left(q \hat{g}_{2}\right)\right) \hat{h}_{4}+\hat{f}_{4} \hat{g}_{2}\left(\partial^{-1}\left(q \hat{h}_{4}\right)\right)+\hat{f}_{1} \hat{g}_{3}\left(\partial^{-1}\left(q \hat{h}_{4}\right)\right) \\
& +\hat{f}_{4}\left(\partial^{-1}\left(w \hat{g}_{3}\right)\right) \hat{h}_{4}+\hat{f}_{3} \hat{g}_{3}\left(\partial^{-1}\left(s \hat{h}_{4}\right)\right)+\hat{f}_{2}\left(\partial^{-1}\left(r \hat{g}_{3}\right)\right) \hat{h}_{4}-\hat{f}_{4}\left(\partial^{-1}\left(s \hat{g}_{4}\right)\right) \hat{h}_{4}+\hat{f}_{2} \hat{g}_{4}\left(\partial^{-1}\left(q \hat{h}_{4}\right)\right) \\
& \left.-\hat{f}_{2}\left(\partial^{-1}\left(q \hat{g}_{4}\right)\right) \hat{h}_{4}+\hat{f}_{4} \hat{g}_{4}\left(\partial^{-1}\left(s \hat{h}_{4}\right)\right)\right\} d x . \\
\beta^{2}: 4 & \int\left\{-r \hat{f}_{4} \hat{g}_{3}\left(\partial^{-1} \hat{h}_{1}\right)+r \hat{f}_{4}\left(\partial^{-1} \hat{g}_{3}\right) \hat{h}_{1}+q \hat{f}_{4} \hat{g}_{4}\left(\partial^{-1} \hat{h}_{1}\right)-r \hat{f}_{3}\left(\partial^{-1} \hat{g}_{4}\right) \hat{h}_{1}+r \hat{f}_{3} \hat{g}_{3}\left(\partial^{-1} \hat{h}_{2}\right)\right. \\
& -r \hat{f}_{4}\left(\partial^{-1} \hat{g}_{3}\right) \hat{h}_{2}-q \hat{f}_{3} \hat{g}_{4}\left(\partial^{-1} \hat{h}_{2}\right)+r \hat{f}_{3} \hat{g}_{4}\left(\partial^{-1} \hat{h}_{2}\right)-r \hat{f}_{4} \hat{g}_{1}\left(\partial^{-1} \hat{h}_{3}\right)+r \hat{f}_{4}\left(\partial^{-1} \hat{g}_{4}\right) \hat{h}_{3}
\end{aligned}
$$


$+q \hat{f}_{4} \hat{g}_{2}\left(\partial^{-1} \hat{h}_{3}\right)-r \hat{f}_{3}\left(\partial^{-1} \hat{g}_{2}\right) \hat{h}_{3}-w \hat{f}_{4} \hat{g}_{3}\left(\partial^{-1} \hat{h}_{3}\right)+r \hat{f}_{2}\left(\partial^{-1} \hat{g}_{3}\right) \hat{h}_{3}-r \hat{f}_{2} \hat{g}_{3}\left(\partial^{-1} \hat{h}_{3}\right)$ $+w \hat{f}_{4}\left(\partial^{-1} \hat{g}_{3}\right) \hat{h}_{3}-r \hat{f}_{2}\left(\partial^{-1} \hat{g}_{3}\right) \hat{h}_{3}+s \hat{f}_{4} \hat{g}_{4}\left(\partial^{-1} \hat{h}_{3}\right)-w \hat{f}_{3}\left(\partial^{-1} \hat{g}_{4}\right) \hat{h}_{3}+q \hat{f}_{2} \hat{g}_{4}\left(\partial^{-1} \hat{h}_{3}\right)$ $-r \hat{f}_{3} \hat{g}_{1}\left(\partial^{-1} \hat{h}_{4}\right)-q \hat{f}_{3}\left(\partial^{-1}\left(\hat{g}_{1}\right) \hat{h}_{4}-q \hat{f}_{3} \hat{g}_{2}\left(\partial^{-1} \hat{h}_{4}\right)-q \hat{f}_{3}\left(\partial^{-1} \hat{g}_{2}\right) \hat{h}_{4}-q \hat{f}_{2}\left(\partial^{-1} \hat{g}_{3}\right) \hat{h}_{4}\right.$ $+w \hat{f}_{2} \hat{g}_{3}\left(\partial^{-1} \hat{h}_{4}\right)-s \hat{f}_{2}\left(\partial^{-1} \hat{g}_{3}\right) \hat{h}_{4}+r \hat{f}_{1} \hat{g}_{3}\left(\partial^{-1} \hat{h}_{4}\right)-s \hat{f}_{3} \hat{g}_{4}\left(\partial^{-1} \hat{h}_{4}+q \hat{f}_{1}\left(\partial^{-1} \hat{g}_{4}\right) \hat{h}_{4}\right)$ $\left.-q \hat{f}_{1} \hat{g}_{4}\left(\partial^{-1} \hat{h}_{4}\right)+s \hat{f}_{3}\left(\partial^{-1} \hat{g}_{4}\right) \hat{h}_{4}\right\} d x$.

$+16 \int\left\{\mathbf{q}\left(\partial^{-1}\left(q \hat{f}_{4}\right)\right)\left(\partial^{-1}\left(r \hat{g}_{3}\right)\right) \hat{h}_{1}-\left(\partial^{-1}\left(r \hat{f}_{3}\right)\right)\left(\partial^{-1}\left(r \hat{g}_{3}\right)\right) \hat{h}_{1}+q\left(\partial^{-1}\left(r \hat{f}_{3}\right)\right) \hat{g}_{3}\left(\partial^{-1}\left(r \hat{h}_{3}\right)\right)\right.$ $\left.-\mathrm{q}\left(\partial^{-1}\left(\mathrm{q} \hat{\mathrm{f}}_{4}\right)\right) \hat{\mathrm{g}}_{3}\left(\partial^{-1}\left(\mathrm{r} \hat{\mathrm{h}}_{1}\right)\right)+\mathrm{q}\left(\partial^{-1} \mathrm{r} \hat{\mathrm{f}}_{3}\right)\left(\partial^{-1} \mathrm{q} \hat{\mathrm{g}}_{4}\right)\right) \hat{\mathrm{h}}_{1}-\mathrm{q}\left(\partial^{-1}\left(\mathrm{q} \hat{\mathrm{f}}_{4}\right)\right)\left(\partial^{-1}\left(\mathrm{q} \hat{\mathrm{g}}_{4}\right)\right) \hat{\mathrm{h}}_{1}$ $\left.+\mathrm{r}\left(\partial^{-1} \mathrm{r} \hat{\mathrm{f}}_{3}\right) \hat{g}_{4}\left(\partial^{-1} \mathrm{r} \hat{\mathrm{h}}_{1}\right)-\mathrm{r}\left(\partial^{-1}\left(\mathrm{r} \hat{\mathrm{f}}_{4}\right)\right) \hat{\mathrm{g}}_{4}\left(\partial^{-1}\left(\mathrm{r} \hat{\mathrm{h}}_{1}\right)\right)-\mathrm{r}\left(\partial^{-1} \mathrm{r} \hat{\mathrm{f}}_{3}\right)\left(\partial^{-1} \mathrm{r} \hat{\mathrm{g}}_{3}\right)\right) \hat{\mathrm{h}}_{2}$ $\left.\left.+r\left(\partial^{-1}\left(q \hat{f}_{4}\right)\right)\left(\partial^{-1} r \hat{g}_{3}\right)\right) \hat{h}_{2}-q\left(\partial^{-1} r \hat{f}_{3}\right)\right) \hat{g}_{3}\left(\partial^{-1}\left(q \hat{h}_{2}\right)\right)+r\left(\partial^{-1}\left(q \hat{f}_{4}\right)\right) \hat{g}_{3}\left(\partial^{-1}\left(q \hat{h}_{2}\right)\right)$ $+r\left(\partial^{-1}\left(r \hat{f}_{3}\right)\right)\left(\partial^{-1}\left(q \hat{g}_{4}\right)\right) \hat{h}_{2}-r\left(\partial^{-1}\left(q \hat{f}_{4}\right)\right)\left(\partial^{-1}\left(q \hat{g}_{4}\right)\right) \hat{h}_{2}-r\left(\partial^{-1}\left(r \hat{f}_{3}\right)\right) \hat{g}_{4}\left(\partial^{-1}\left(q \hat{h}_{2}\right)\right)$ $\left.+r\left(\partial^{-1}\left(q \hat{f}_{4}\right)\right) \hat{g}_{4}\left(\partial^{-1}\left(q \hat{h}_{2}\right)\right)-q\left(\partial^{-1} r \hat{f}_{3}\right)\right)\left(\partial^{-1}\left(r \hat{g}_{1}\right)\right) \hat{h}_{3}+q\left(\partial^{-1}\left(q \hat{f}_{4}\right)\right)\left(\partial^{-1}\left(r \hat{g}_{1} \hat{h}_{3}\right)\right)$ $\left.+q\left(\partial^{-1}\left(r \hat{f}_{3}\right)\right) \hat{g}_{1}\left(\partial^{-1}\left(r \hat{h}_{3}\right)\right)-q\left(\partial^{-1}\left(q \hat{f}_{4}\right)\right) \hat{g}_{1}\left(\partial^{-1}\left(r \hat{h}_{3}\right)\right)+q\left(\partial^{-1} r \hat{f}_{3}\right)\right)\left(\partial^{-1}\left(q \hat{g}_{2}\right)\right) \hat{h}_{3}$ $-q\left(\partial^{-1}\left(q \hat{f}_{4}\right)\right)\left(\partial^{-1}\left(q \hat{g}_{2}\right)\right) \hat{h}_{3}+r\left(\partial^{-1}\left(r \hat{f}_{3}\right)\right) \hat{g}_{2}\left(\partial^{-1}\left(r \hat{h}_{3}\right)\right)-r\left(\partial^{-1}\left(q \hat{f}_{4}\right)\right) \hat{g}_{2}\left(\partial^{-1}\left(r \hat{h}_{3}\right)\right)$ $\left.-\mathrm{q}\left(\partial^{-1}\left(\mathrm{r} \hat{\mathrm{f}}_{3}\right)\right)\left(\partial^{-1}\left(w \hat{\mathrm{g}}_{3}\right)\right) \hat{\mathrm{h}}_{3}+\mathrm{q}\left(\partial^{-1}\left(\mathrm{q} \hat{\mathrm{f}}_{4}\right)\right)\left(\partial^{-1}\left(w \hat{\mathrm{g}}_{3}\right)\right) \hat{\mathrm{h}}_{3}+\mathrm{q}\left(\partial^{-1} \mathrm{r} \hat{\mathrm{f}}_{1}\right)\right) \hat{\mathrm{g}}_{3}\left(\partial^{-1}\left(\mathrm{r} \hat{\mathrm{h}}_{3}\right)\right)$ $-\mathrm{q}\left(\partial^{-1}\left(\mathrm{q} \hat{\mathrm{f}}_{2}\right)\right) \hat{\mathrm{g}}_{3}\left(\partial^{-1}\left(\mathrm{r} \hat{\mathrm{h}}_{3}\right)\right)+\mathrm{q}\left(\partial^{-1}\left(w \hat{\mathrm{f}}_{3}\right)\right) \hat{\mathrm{g}}_{3}\left(\partial^{-1}\left(\mathrm{r} \hat{\mathrm{h}}_{3}\right)\right)+\mathrm{s}\left(\partial^{-1}\left(\mathrm{r} \hat{\mathrm{f}}_{3}\right)\right) \hat{\mathrm{g}}_{3} \partial^{-1}\left(\mathrm{r} \hat{\mathrm{h}}_{3}\right)$ $\left.-\mathrm{q}\left(\partial^{-1}\left(s \hat{f}_{4}\right)\right) \hat{g}_{3}\left(\partial^{-1} r \hat{h}_{3}\right)-s\left(\partial^{-1}\left(q \hat{f}_{3}\right)\right) \hat{g}_{3}\left(\partial^{-1}\left(r \hat{h}_{3}\right)\right)-\mathrm{q}\left(\partial^{-1} r \hat{f}_{1}\right)\left(\partial^{-1}\left(r \hat{g}_{3}\right)\right) \hat{h}_{3}\right)$ $+\mathrm{q}\left(\partial^{-1}\left(\mathrm{q} \hat{\mathrm{f}}_{2}\right)\right)\left(\partial^{-1}\left(\mathrm{r} \hat{g}_{3}\right)\right) \hat{\mathrm{h}}_{3}-\mathrm{q}\left(\partial^{-1}\left(w \hat{\mathrm{f}}_{3}\right)\right)\left(\partial^{-1}\left(\mathrm{r} \hat{\mathrm{g}}_{3}\right)\right) \hat{\mathrm{h}}_{3}-\mathrm{s}\left(\partial^{-1}\left(\mathrm{r} \hat{\mathrm{f}}_{3}\right)\right)\left(\partial^{-1}\left(\mathrm{r} \hat{\mathrm{g}}_{3}\right)\right) \hat{\mathrm{h}}_{3}$ $+\mathrm{q}\left(\partial^{-1}\left(\mathrm{~s} \hat{\mathrm{f}}_{4}\right)\right)\left(\partial^{-1}\left(\mathrm{rg} \hat{\mathrm{g}}_{3}\right)\right) \hat{\mathrm{h}}_{3}+\mathrm{s}\left(\partial^{-1}\left(\mathrm{q} \hat{\mathrm{f}}_{4}\right)\right)\left(\partial^{-1}\left(\mathrm{r} \hat{\mathrm{g}}_{3}\right)\right) \hat{\mathrm{h}}_{3}+\mathrm{q}\left(\partial^{-1} \mathrm{r} \hat{\mathrm{f}}_{3}\right) \hat{\mathrm{g}}_{3}\left(\partial^{-1}\left(w \hat{\mathrm{h}}_{3}\right)\right)$ $-q\left(\partial^{-1}\left(q \hat{f}_{4}\right)\right) \hat{g}_{3}\left(\partial^{-1}\left(w \hat{h}_{3}\right)\right)+r\left(\partial^{-1} r \hat{f}_{1}\right) \hat{g}_{3}\left(\partial^{-1}\left(r \hat{h}_{3}\right)\right)-r\left(\partial^{-1}\left(q \hat{f}_{2}\right)\right) \hat{g}_{3}\left(\partial^{-1}\left(r \hat{h}_{3}\right)\right)$ $+r\left(\partial^{-1}\left(w \hat{f}_{3}\right)\right) \hat{g}_{4}\left(\partial^{-1}\left(r \hat{h}_{3}\right)\right)+w\left(\partial^{-1}\left(r \hat{f}_{3}\right)\right) \hat{g}_{3}\left(\partial^{-1}\left(r \hat{h}_{3}\right)\right)-r\left(\partial^{-1}\left(s \hat{f}_{4}\right)\right) \hat{g}_{3}\left(\partial^{-1}\left(r \hat{h}_{3}\right)\right)$ $\left.-w\left(\partial^{-1}\left(q \hat{f}_{4}\right)\right) \hat{g}_{3}\left(\partial^{-1}\left(r \hat{h}_{3}\right)\right)+q\left(\partial^{-1} r \hat{f}_{3}\right)\right)\left(\partial^{-1}\left(s \hat{g}_{4}\right)\right) \hat{h}_{3}-q\left(\partial^{-1}\left(q \hat{f}_{4}\right)\right)\left(\partial^{-1}\left(s \hat{g}_{3}\right)\right) \hat{h}_{3}$ $+\mathrm{r}\left(\partial^{-1} \mathrm{r} \hat{\mathrm{f}}_{3}\right) \hat{\mathrm{g}}_{4}\left(\partial^{-1}\left(w \hat{\mathrm{h}}_{3}\right)\right)-\mathrm{r}\left(\partial^{-1}\left(\mathrm{q} \hat{\mathrm{f}}_{4}\right)\right) \hat{\mathrm{g}}_{4}\left(\partial^{-1}\left(w \hat{\mathrm{h}}_{3}\right)\right)+\mathrm{q}\left(\partial^{-1} \mathrm{r} \hat{\mathrm{f}}_{1}\right)\left(\partial^{-1}\left(\mathrm{q} \hat{\mathrm{g}}_{4}\right)\right) \hat{\mathrm{h}}_{3}$ $-q\left(\partial^{-1}\left(q \hat{f}_{2}\right)\right)\left(\partial^{-1}\left(q \hat{g}_{4}\right)\right) \hat{h}_{3}+q\left(\partial^{-1}\left(w \hat{f}_{3}\right)\right)\left(\partial^{-1}\left(q \hat{g}_{4}\right)\right) \hat{h}_{3}+s\left(\partial^{-1}\left(r \hat{f}_{3}\right)\right)\left(\partial^{-1}\left(r \hat{g}_{4}\right)\right) \hat{h}_{3}$ $-q\left(\partial^{-1}\left(s \hat{f}_{4}\right)\right)\left(\partial^{-1}\left(q \hat{g}_{4}\right)\right) \hat{h}_{3}-s\left(\partial^{-1}\left(q \hat{f}_{4}\right)\right)\left(\partial^{-1}\left(q \hat{g}_{4}\right)\right) \hat{h}_{3}-r\left(\partial^{-1}\left(r \hat{f}_{3}\right)\right)\left(\partial^{-1}\left(r \hat{g}_{1}\right)\right) \hat{h}_{4}$ $+r\left(\partial^{-1}\left(q \hat{f}_{3}\right)\right)\left(\partial^{-1}\left(r \hat{g}_{3}\right)\right) \hat{h}_{3}-q\left(\partial^{-1}\left(r \hat{f}_{3}\right)\right) \hat{g}_{1}\left(\partial^{-1}\left(q \hat{h}_{4}\right)\right)+q\left(\partial^{-1}\left(q \hat{f}_{4}\right)\right) \hat{g}_{3}\left(\partial^{-1}\left(q \hat{h}_{4}\right)\right)$ $+r\left(\partial^{-1}\left(r \hat{f}_{3}\right)\right)\left(\partial^{-1}\left(q \hat{g}_{2}\right)\right) \hat{h}_{4}-r\left(\partial^{-1}\left(q \hat{f}_{4}\right)\right)\left(\partial^{-1}\left(q \hat{g}_{2}\right)\right) \hat{h}_{4}-r\left(\partial^{-1} r \hat{f}_{3}\right) \hat{g}_{2}\left(\partial^{-1}\left(q \hat{h}_{4}\right)\right)$ $\left.+r\left(\partial^{-1}\left(q \hat{f}_{4}\right)\right) \hat{g}_{2}\left(\partial^{-1}\left(q \hat{h}_{4}\right)\right)-q\left(\partial^{-1} r \hat{f}_{1}\right)\right) \hat{g}_{3}\left(\partial^{-1}\left(q \hat{h}_{3}\right)\right)+q\left(\partial^{-1}\left(q \hat{f}_{2}\right)\right) \hat{g}_{3}\left(\partial^{-1}\left(q \hat{h}_{4}\right)\right)$ $\left.-q\left(\partial^{-1}\left(w \hat{f}_{3}\right)\right) \hat{g}_{3}\left(\partial^{-1}\left(q \hat{h}_{4}\right)\right)-s\left(\partial^{-1}\left(r \hat{f}_{3}\right)\right) \hat{g}_{3} \partial^{-1}\left(q \hat{h}_{4}\right)\right)+q\left(\partial^{-1}\left(s \hat{f}_{4}\right)\right) \hat{g}_{3}\left(\partial^{-1} q \hat{h}_{4}\right)$ $+s\left(\partial^{-1}\left(q \hat{f}_{4}\right)\right) \hat{g}_{3}\left(\partial^{-1}\left(q \hat{h}_{4}\right)\right)-r\left(\partial^{-1}\left(r \hat{f}_{3}\right)\right)\left(\partial^{-1}\left(w \hat{g}_{3}\right)\right) \hat{h}_{4}+r\left(\partial^{-1}\left(q \hat{f}_{4}\right)\right)\left(\partial^{-1}\left(w \hat{g}_{3}\right)\right) \hat{h}_{4}$ $\left.-q\left(\partial^{-1}\left(r \hat{f}_{3}\right)\right) \hat{g}_{3}\left(\partial^{-1}\left(s \hat{h}_{4}\right)\right)+q\left(\partial^{-1}\left(q \hat{f}_{4}\right)\right) \hat{g}_{3}\left(\partial^{-1}\left(s \hat{h}_{4}\right)\right)+r\left(\partial^{-1} r \hat{f}_{1}\right)\right)\left(\partial^{-1}\left(r \hat{g}_{3}\right)\right) \hat{h}_{4}$ $+\mathrm{r}\left(\partial^{-1}\left(\mathrm{q} \hat{\mathrm{f}}_{2}\right)\right)\left(\partial^{-1}\left(\mathrm{rg} \hat{g}_{3}\right)\right) \hat{\mathrm{h}}_{4}-\mathrm{r}\left(\partial^{-1}\left(w \hat{\mathrm{f}}_{3}\right)\right)\left(\partial^{-1}\left(\mathrm{r} \hat{g}_{3}\right)\right) \hat{\mathrm{h}}_{4}-w\left(\partial^{-1}\left(\mathrm{r} \hat{\mathrm{f}}_{3}\right)\right)\left(\partial^{-1}\left(\mathrm{r} \hat{g}_{3}\right)\right) \hat{\mathrm{h}}_{4}$ $+r\left(\partial^{-1}\left(s \hat{f}_{4}\right)\right)\left(\partial^{-1}\left(r \hat{g}_{3}\right)\right) \hat{h}_{4}+w\left(\partial^{-1}\left(q \hat{f}_{4}\right)\right)\left(\partial^{-1}\left(r \hat{g}_{3}\right)\right) \hat{h}_{4}+r\left(\partial^{-1} r \hat{f}_{3}\right)\left(\partial^{-1}\left(s \hat{g}_{4}\right)\right) \hat{h}_{4}$ $-r\left(\partial^{-1}\left(q \hat{f}_{4}\right)\right)\left(\partial^{-1}\left(s \hat{g}_{4}\right)\right) \hat{h}_{4}-r\left(\partial^{-1}\left(r \hat{f}_{1}\right)\right) \hat{g}_{4}\left(\partial^{-1}\left(q \hat{h}_{4}\right)\right)+r\left(\partial^{-1}\left(q \hat{f}_{2}\right)\right) \hat{g}_{4}\left(\partial^{-1}\left(q \hat{h}_{4}\right)\right)$ $-r\left(\partial^{-1}\left(w \hat{f}_{3}\right)\right) \hat{g}_{4}\left(\partial^{-1}\left(q \hat{h}_{4}\right)\right)-w\left(\partial^{-1}\left(r \hat{f}_{3}\right)\right) \hat{g}_{4}\left(\partial^{-1}\left(q \hat{h}_{4}\right)\right)+r\left(\partial^{-1}\left(s \hat{f}_{4}\right)\right) \hat{g}_{4}\left(\partial^{-1}\left(q \hat{h}_{4}\right)\right)$ $\left.+w\left(\partial^{-1}\left(q \hat{f}_{4}\right)\right) \hat{g}_{4}\right)\left(\partial^{-1}\left(q \hat{h}_{4}\right)+r\left(\partial^{-1} r \hat{f}_{1}\right)\left(\partial^{-1}\left(q \hat{g}_{4}\right)\right) \hat{h}_{4}-r\left(\partial^{-1}\left(q \hat{f}_{2}\right)\right)\left(\partial^{-1}\left(q \hat{g}_{4}\right)\right) \hat{h}_{4}\right.$ $+r\left(\partial^{-1}\left(w \hat{f}_{3}\right)\right)\left(\partial^{-1}\left(q \hat{g}_{4}\right)\right) \hat{h}_{4}+w\left(\partial^{-1}\left(r \hat{f}_{3}\right)\right)\left(\partial^{-1}\left(q \hat{g}_{4}\right)\right) \hat{h}_{4}-r\left(\partial^{-1}\left(s \hat{f}_{4}\right)\right)\left(\partial^{-1}\left(q \hat{g}_{4}\right)\right) \hat{h}_{4}$ $\left.-w\left(\partial^{-1}\left(q \hat{f}_{4}\right)\right)\left(\partial^{-1}\left(q \hat{g}_{4}\right)\right) \hat{h}_{4}-r\left(\partial^{-1} r \hat{f}_{3}\right) \hat{g}_{4}\left(\partial^{-1}\left(s \hat{h}_{4}\right)\right)+r\left(\partial^{-1}\left(q \hat{f}_{4}\right)\right) \hat{g}_{4}\left(\partial^{-1}\left(q \hat{h}_{4}\right)\right)\right\} d x$. 
Through a direct calculation, we obtain that above three sums with a cycle of $\hat{f}, \hat{g}$, and $\hat{h}$ are all equal to zero.

\section{Acknowledgment}

This work was supported by the Nature Science Foundation of Shandong Province of China (Grant No. ZR2014AM001). The authors are very grateful to the editors and the referees for their helpful suggestions.

\section{References}

[1] B. Fuchssteiner, Coupling of completely integrable systems: the perturbation bundle, Applications of analytic and geometric methods to nonlinear differential equations, Exeter, (1992) NATO Adv. Sci. Inst. Ser. C Math. Phys. Sci., Kluwer Acad. Publ., Dordrecht, 413 (1993), 125-138. 1

[2] C.-H. Gu, H.-S. Hu, Z.-X. Zhou, Darboux transformation in soliton theory and its geometric applications, Shanghai Scientific and Technical Publishers, Shanghai, (1999). 1, 4

[3] D. B. Hinton, A. K. Jordan, M. Klaus, J. K. Shaw, Inverse scattering on the line for a Dirac system, J. Math. Phys., 32 (1991), 3015-3030. 1, 1

[4] W.-X. Ma, Some problems in nonlinear integrable systems, Ph.D. Thesis, Computing Center of the Chinese Academy of Sciences, China, (1990). 3

[5] W.-X. Ma, Binary nonlinearization for the Dirac systems, A Chinese summary appears in Chinese Ann. Math. Ser. A, 18 (1997), 134, Chinese Ann. Math. Ser. B, 18 (1997), 79-88. 1

[6] W.-X. Ma, Integrable couplings of soliton equations by perturbations. I. A general theory and application to the KdV hierarchy, Methods Appl. Anal., 7 (2000), 21-56. 1

[7] W.-X. Ma, Enlarging spectral problems to construct integrable couplings of soliton equations, Phys. Lett. A, 316 (2003), $72-76$.

[8] W.-X. Ma, Loop algebras and bi-integrable couplings, Chin. Ann. Math. Ser. B, 33 (2012), 207-224.

[9] W.-X. Ma, M. Chen, Hamiltonian and quasi-Hamiltonian structures associated with semi-direct sums of Lie algebras, J. Phys. A, 39 (2006), 10787-10801. 3

[10] W.-X. Ma, B. Fuchssteiner, Integrable theory of the perturbation equations, Chaos Solitons Fractals, 7 (1996), 1227-1250. 1

[11] W.-X. Ma, J.-H. Meng, H.-Q. Zhang, Tri-integrable couplings by matrix loop algebras, Int. J. Nonlinear Sci. Numer. Simul., 14 (2013), 377-388.

[12] W.-X. Ma, X.-X. Xu, Y.-F. Zhang, Semi-direct sums of Lie algebras and continuous integrable couplings, Phys. Lett. A, 351 (2006), 125-130. 1

[13] W.-X. Ma, R.-G. Zhou, A coupled AKNS-Kaup-Newell soliton hierarchy, J. Math. Phys., 40 (1999), 4419-4428. 1, 1

[14] F. Magri, A simple model of the integrable Hamiltonian equation, J. Math. Phys., 19 (1978), 1156-1162. 3

[15] F. Magri, A geometrical approach to the nonlinear solvable equations, Nonlinear evolution equations and dynamical systems, Proc. Meeting, Univ. Lecce, Lecce, (1979), Lecture Notes in Phys., Springer, Berlin-New York, 120 (1980), 233-263. 1, 1, 3

[16] G. Neugebauer, R. Meinel, General N-soliton solution of the AKNS class on arbitrary background, Phys. Lett. A, 100 (1984), 467-470. 1, 4

[17] P. J. Olver, Applications of Lie groups to differential equations, Graduate Texts in Mathematics, Springer-Verlag, New York, (1986). 1, 1, 3, 3

[18] K. M. Tamizhmani, M. Lakshmanan, Complete integrability of the Korteweg-de Vries equation under perturbation around its solution: Lie-Bäcklund symmetry approach, J. Phys. A, 16 (1983), 3773-3782. 1

[19] G. Z. Tu, The trace identity, a powerful tool for constructing the Hamiltonian structure of integrable systems, J. Math. Phys., 30 (1989), 330-338. 1

[20] X.-X. Xu, An integrable coupling hierarchy of the $M K d V$ _integrable systems, its Hamiltonian structure and corresponding nonisospectral integrable hierarchy, Appl. Math. Comput., 216 (2010), 344-353. 1 